\title{
CONTINUUM LIMIT OF THE NONLOCAL $p$-LAPLACIAN EVOLUTION PROBLEM ON RANDOM INHOMOGENEOUS GRAPHS
}

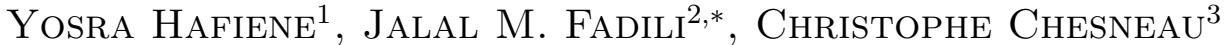 \\ AND ABDERRAhim ElmoATAZ ${ }^{4}$
}

\begin{abstract}
In this paper we study numerical approximations of the evolution problem governed by the nonlocal $p$-Laplacian operator with a given kernel and homogeneous Neumann boundary conditions. More precisely, we consider discretized versions on inhomogeneous random graph sequences, establish their continuum limits and provide error bounds with nonasymptotic rate of convergence of solutions of the discrete problems to their continuum counterparts as the number of vertices grows. Our bounds reveal the role of the different parameters that come into play, and in particular that of $p$ and of the geometry/regularity of the initial data and the kernel.
\end{abstract}

Mathematics Subject Classification. 35A35, 65N12, 65N15, 41A17, $05 \mathrm{C} 80$.

Received April 26, 2019. Accepted August 31, 2019.

\section{INTRODUCTION}

\subsection{Problem statement}

Our main goal in this paper is to study numerical approximations on random inhomogeneous graphs to a nonlocal nonlinear diffusion problem, involving the nonlocal $p$-Laplacian operator with homogeneous Neumann boundary conditions. More precisely, the nonlocal $p$-Laplacian evolution problem with Neumann boundary conditions that we deal with is

$$
\begin{cases}\frac{\partial}{\partial t} u(x, t)=-\Delta_{p}^{K}(u(x, t)), & x \in \Omega, t>0 \\ u(x, 0)=g(x), & x \in \Omega\end{cases}
$$

where

$$
\boldsymbol{\Delta}_{p}^{K}(u(x, t))=-\int_{\Omega} K(x, y)|u(y, t)-u(x, t)|^{p-2}(u(y, t)-u(x, t)) \mathrm{d} y
$$

Keywords and phrases. Nonlocal diffusion, p-Laplacian, inhomogeneous random graphs, graph limits, graphon, numerical approximation.

1 Normandie Univ, ENSICAEN, UNICAEN, CNRS, GREYC, Caen, France.

2 Normandie Univ, ENSICAEN, CNRS, GREYC, Caen, France.

3 Normandie Univ, UNICAEN, CNRS, LMNO, Caen, France.

4 Normandie Univ, UNICAEN, CNRS, GREYC, Caen, France.

*Corresponding author: Jalal.Fadili@greyc.ensicaen.fr 
where $\Omega \subset \mathbb{R}$ a bounded domain, and without loss of generality we take $\Omega=[0,1]^{5}$. The kernel $K \in L^{\infty}\left(\Omega^{2}\right)$ is a symmetric measurable and nonnegative mapping. Throughout the paper, we will assume that $p \in] 1,+\infty[$. Existence and uniqueness of a strong solution to $(\mathcal{P})$ in the space $L^{p}(\Omega)$ was shown in Theorem 3.1 of [17] (relying on arguments from [2]).

The nonlocal $p$-Laplacian operator appears naturally in the study of many nonlocal diffusion phenomena. It arises for instance in a number of applications such as continuum mechanics, phase transition phenomena, population dynamics, image processing and game theory (see $[1,2,16,20]$ and the references therein). On the other hand, recently, there has been a high interest in adapting and applying discretized versions of PDEs such as $(\mathcal{P})$ on data defined on arbitrary graphs and networks. Given the discrete nature of data in practice, graphs constitute a natural structure suited to their representation. The demand for such methods is motivated by existing and potential future applications, such as in machine learning and mathematical image/signal/data processing (see among other references $[8,10,11,15]$ ). Indeed, any kind of discrete data can be represented by a graph in an abstract form in which the vertices are associated to the data and the edges correspond to relationships within the data. These practical considerations naturally lead to a discrete time and space approximation of $(\mathcal{P})$.

To do this, fix $n \in \mathbb{N}^{*}$. Let $G_{n}=\left(V\left(G_{n}\right), E\left(G_{n}\right)\right)$, where $V\left(G_{n}\right)$ stands for the set of nodes and $E\left(G_{n}\right) \subset$ $V\left(G_{n}\right) \times V\left(G_{n}\right)$ denotes the edges set, be a sequence of simple graphs, i.e. undirected graphs without loops and parallel edges.

Next, we consider the fully discrete counterpart of $(\mathcal{P})$ on a graph $G_{n}$ using the forward Euler scheme. For that, let us consider a partition (not necessarily uniform) of the time interval $[0, T]$ into intervals of sizes $\left\{\tau_{h}\right\}_{h=1}^{N}, N \in \mathbb{N}^{*}$, and denote $\tau=\max _{h \in[N]} \tau_{h}$, where $[N] \stackrel{\text { def }}{=}\{1, \ldots, N\}$ for any integer $N$. Denote $u_{i}^{h} \stackrel{\text { def }}{=} u\left(x_{i}, t_{h}\right)$ and $g_{i} \stackrel{\text { def }}{=} g\left(x_{i}\right)$. Then for $h \in[N]$, consider

$$
\left\{\begin{array}{l}
\frac{u_{i}^{h}-u_{i}^{h-1}}{\tau_{h}}=\frac{1}{n} \sum_{j:(i, j) \in E\left(G_{n}\right)}\left|u_{j}^{h-1}-u_{i}^{h-1}\right|^{p-2}\left(u_{j}^{h-1}-u_{i}^{h-1}\right), \\
u_{i}^{0}=g_{i}, \quad i \in\{1, \ldots, n\}
\end{array}\right.
$$

Thus, $\left(\mathcal{P}_{n, \tau}^{d}\right)$ induces a discrete diffusion process parametrized by the structure of the graph whose adjacency matrix captures the (nonlocal) interactions. As such, it can be viewed as a discrete approximation of a continuum problem such as $(\mathcal{P})$.

Several questions then naturally arise:

- Does the discrete problem $\left(\mathcal{P}_{n, \tau}^{d}\right)$, and in what sense, has a continuum limit $($ as $n \rightarrow+\infty)$ ?

- What is the rate of convergence to this limit? Is this limit consistent/related with the unique strong solution of $(\mathcal{P})$ ?

- What are the parameters involved in this rate and what is their influence on the convergence rate?

This paper provides answers to these questions for graphs drawn from a random inhomogeneous model introduced by [3]. The "classical" random graph models, in particular dense graphs, are "homogeneous", in the sense that the nodes degrees tend to be concentrated around a typical value, so that all vertices are exactly equivalent in the definition of the model. Furthermore, in a typical realization, most vertices are in some sense similar to most others. In contrast, some graphs arising in real-world applications do not have this property and are inhomogeneous. One reason is that the vertices may have been "born" at different times, with old and new vertices having very different properties. Thus, there has been a lot of recent interest in defining and studying networks on inhomogeneous random graph models (see Sect. 2 for further details). Our aim is to investigate this graph model and study the continuum limit of the $p$-Laplacian discrete approximation on these random graph models that can be dense or sparse.

\footnotetext{
${ }^{5}$ Only boundedness of $\Omega$ is actually needed but we take $\Omega$ as a closed set as well to conform to our setting of graphs. Moreover, though we here focus on the one-dimensional case $\Omega \subset \mathbb{R}$, several of our results can be extended to higher dimension.
} 


\subsection{Contributions}

In [17], we provided a rigorous justification of the continuum limit $(\mathcal{P})$ for the discrete $p$-Laplacian on deterministic dense graphs (graphs with $n$ vertices and $\Theta\left(n^{2}\right)$ edges ${ }^{6}$ ). The analysis of the continuum limit in [17] uses ideas from the theory of dense graph limits $[6,21,22]$, which for every convergent family of dense graphs defines the limiting object, a measurable symmetric bounded and nonnegative function $K$ called graphon (see Sect. 2 for a brief overview on graphons). This object characterizes the completion of the space of all graphs with respect to an appropriate metric. In [17], for convergent sequences of deterministic dense graphs $\left\{G_{n}\right\}_{n \in \mathbb{N}}$, it was shown that with the kernel in $(\mathcal{P})$ taken to be the graphon associated to $\left\{G_{n}\right\}_{n \in \mathbb{N}^{*}}$, the solution of $(\mathcal{P})$ is well-approximated by those of the totally discrete problems $\left(\mathcal{P}_{n, \tau}^{d}\right)$. We gave precise convergence rates as a function of $n$ the discretization time step $\tau$.

However, the analysis in [17] does not deal with inhomogeneous graphs, see [3], which allow for sparse (but not too sparse) graphs with $o\left(n^{2}\right)$ but $\omega(n)$ edges. It does not either exhibit the typical error bounds achieved over a sequence of (random) graphs drawn from this inhomogeneous graph model. The main concern of this paper is to bridge this gap by studying continuum limits of $\left(\mathcal{P}_{n, \tau}^{d}\right)$ on inhomogeneous random graphs.

Combining tools from evolution equations, random graph theory and deviation inequalities, we establish nonasymptotic rate of convergence of the discrete solution to its continuum limit with a controlled (high) probability. More precisely, we start by considering the case of random graph models generated by a deterministic sequence of nodes. We prove nonasymptotic error bounds that hold with a controlled probability (Thm. 3.3). These results serve as a basis to deal with the totally random graph model, i.e. where both the nodes and edges are random (Thm. 3.6). In turn, this shows convergence of solutions of the discrete models to the solution of the continuum problem as the number of vertices $n$ grows. To get the corresponding convergence rate, we additionally assume that the kernel $K$ and the initial data $g$ belong to the versatile class Lipschitz spaces $\operatorname{Lip}\left(s, L^{q}\left(\Omega^{2}\right)\right)$ and $\operatorname{Lip}\left(s^{\prime}, L^{q}(\Omega)\right)$. Roughly speaking, $\operatorname{Lip}\left(s, L^{q}\left(\Omega^{2}\right)\right)$ contains functions with $s$ "derivatives" in $L^{q}\left(\Omega^{2}\right)$. They contain in particular functions of bounded variation and those of fractal structure for appropriate values of $s$, see (see Appendix A for a brief introduction to these functional spaces). Using in addition arguments from approximation theory on these spaces, we reveal the influence of the value of $p$, the density of the graph, the regularity of the graphon $K$ and that of the initial data $g$ both on the convergence rate and the probability of success. In particular, we isolate different regimes where the rate exhibits different scalings.

\subsection{Relation to prior work}

In [25] and earlier [24], the author studied convergence of discrete approximations of a nonlinear heat equation governed by a Lipschitz continuous potential, first on dense deterministic graphs and then on dense random ones, without discretization of time. However, though the work of [25] was important to us, it differs markedly from ours in many crucial aspects. Indeed, we use some standard arguments from numerical analysis of evolution problems but also specific and sophisticated ones tied to the $p$-Laplacian. Typically, well-posedness and Lipschitz continuity of the solutions w.r.t. to the kernel and initial data for the evolution problem with the $p$-Laplacian is much harder to establish than for the problem considered in [24,25] (see [17]). Second, comparing [25] and our current work, we use completely different paths to prove consistency in the random case. Indeed, while the claim in [25] is asymptotic by nature as it completely relies on application of the central limit theorem (CLT), the latter argument cannot be applied to our evolution problem (except for the trivial case $p=2$ ). Rather, we establish a nonasymptotic deviation inequality, both in the partly and completely random graph model, relying on a careful control of a random process using sharp inequalities from probability theory (Rosenthal and Bernstein, see Lem. A.1). Thus, we are able to provide the probability of success of our bound for fixed $n$ and we exhibit the dependence of both the error bound and the probability on the problem parameters $(p, T$, graph model, kernel $K$, initial data $g$ ). This is in a stark contrast to the asymptotic claims in [25].

In [19], the authors extended the analysis of [25] to sparse random graphs corresponding to $L^{2}\left(\Omega^{2}\right)$ graphons and proved almost sure consistency. While a first version of this paper was under review, we also became aware

\footnotetext{
${ }^{6}$ We recall the usual Landau asymptotic notations: $O(),. \Theta(),. o($.$) and \omega($.$) .$
} 
of the recent preprint [26] which studied the Kuramoto model on a sequence of converging dense and sparse graph sequences. It proved almost sure convergence of the discrete problems on such graphs to continuum limit with time intervals of size $T=O(\log (n))$. In addition to the fact that our evolution problem is different and more intricate, our random graph model is different from that of $[19,26]$. Both models allow for sparse graphs, but ours only for those with $o\left(n^{2}\right)$ but $\omega(n)$ edges with bounded graphons, while theirs covers graphs with $O(n)$ edges and $L^{q}\left(\Omega^{2}\right)$ graphons. Whether our results on the $p$-Laplacian can be extended to such sparse graphs is an open problem. In fact, even well-posedness (existence and uniqueness) of the $p$-Laplacian evolution problem $(\mathcal{P})$ with unbounded kernels $K$ remains completely open in the literature. Our results can also cope with time intervals $T=O(\log (n))$ as discussed in Remark 3.4(v). Observe finally that the convergence claim of [19] is asymptotic (almost sure convergence), relying on the standard Markov inequality and Borel-Cantelli lemma, while ours are nonasymptotic with a precise rate and probability of success.

\subsection{Paper organization}

The rest of the paper is organized as follows. In Section 2, we provide some pre-requisites on graph limits and graphons and then define our inhomogeneous $K$-random graph model that we deal with throughout the paper. We also specify the assumptions needed to get our results and give a class of graphs for which our assumptions hold true. Section 3 is devoted to the main result of the paper. We begin our analysis by treating random graph sequences generated by deterministic nodes in Section 3.1. Then, in Section 3.2 we consider the general model defined previously in Section 2. After getting the convergence of the discrete model to its continuum limit and identifying the corresponding rate, in Section 3.3, we discuss the different regimes of the convergence rate as a function of the problem parameters. Some technical material is deferred to Appendix A.

\subsection{Notations}

For a given vector $u=\left(u_{1}, \ldots, u_{n}\right)^{\top} \in \mathbb{R}^{n}$, we define the norm $\|\cdot\|_{p, n}$

$$
\|u\|_{p, n}=\left(\frac{1}{n} \sum_{i=1}^{n}\left|u_{i}\right|^{p}\right)^{\frac{1}{p}} .
$$

For an integer $n \in \mathbb{N}^{*}$, we denote $[n]=\{1, \ldots, n\}$. For any set $S, \bar{S}$ is its closure and $|S|$ is its cardinality or its Lebesgue measure (to be understood from the context). $\chi_{S}$ is the characteristic function of the set $S$ (takes 1 in it and 0 otherwise).

$C\left(0, T ; L^{p}(\Omega)\right)$ denotes the space of uniformly time continuous functions with values in $L^{p}(\Omega)$. We endow this space with the norm

$$
\|u\|_{C\left(0, T ; L^{p}(\Omega)\right)} \stackrel{\text { def }}{=} \sup _{t \in[0, T]}\|u(t)\|_{L^{p}(\Omega)} .
$$

For $d \in\{1,2\}, \operatorname{Lip}\left(s, L^{q}\left(\Omega^{d}\right)\right)$ is the Lipschitz space which consists of functions with, roughly speaking, $s$ "derivatives" in $L^{q}\left(\Omega^{d}\right)([9]$, Ch. 2, Sect. 9). Only values $\left.s \in] 0,1\right]$ are of interest to us. See Section A.2 for further details on these spaces and approximation theoretic results on them.

\section{THE RANDOM INHOMOGENEOUS GRAPH MODEL}

\subsection{Graph limits and graphons}

We start with some important results from the theory of graph limits that will be crucial to our exposition. The theory of graph limits was introduced by Lovász and Szegedy in 2006 [22] and then further developed in a series of papers (see the book [21] for a comptrehensive bibliography). A key goal of Lovász and Szegedy was to understand large graph structures by characterizing convergence for sequences of graphs which grow unboundedly, thereby constructing a natural limit object. 
Let $G_{n}=\left(V\left(G_{n}\right), E\left(G_{n}\right)\right), n \in \mathbb{N}^{*}$, be a sequence of finite and simple graphs. Every finite simple graph $G_{n}$ such that $V\left(G_{n}\right)=[n]$ can be represented by a measurable function $K_{G_{n}}: \Omega^{2} \rightarrow \Omega$ called a pixel kernel. Its construction is as follows: split the interval $\Omega$ into $n$ equal intervals, and for every $(x, y) \in\left[\frac{i-1}{n}, \frac{i}{n}\left[\times\left[\frac{j-1}{n}, \frac{j}{n}[\right.\right.\right.$, define

$$
K_{G_{n}}(x, y)= \begin{cases}1 & \text { if }(i, j) \in E\left(G_{n}\right) \\ 0 & \text { otherwise. }\end{cases}
$$

For weighted graphs with edge weights $\{\beta(i, j)\}_{(i, j) \in V(G)^{2}}$, the pixel kernel $K_{G_{n}}$ becomes

$$
K_{G_{n}}(x, y)= \begin{cases}\beta(i, j) & \text { if }(i, j) \in E\left(G_{n}\right), \\ 0 & \text { otherwise. }\end{cases}
$$

This construction is not unique, however given a graph, the set of pixel kernels arising via (2.1) can be considered to be equivalent via the weakly isomorphic relation.

Convergent graph sequences $\left\{G_{n}\right\}_{n \in \mathbb{N}^{*}}$ have a limit object, which can be represented as a measurable and symmetric function $K: \Omega^{2} \rightarrow[0,1]$ called graphon, and the function $K$ is uniquely determined up to measurepreserving transformation; see Theorem 2.1 of [7]. Intuitively, a graphon can be thought of as a generalization of the adjacency matrix of a (weighted) graph which has a continuum number of vertices. Actually, the space of graphons is the the completion of the metric space of graphs, or equivalently pixel kernels, relative to the so-called cut distance; see Theorem 2.6 of [6]. Conversely, Theorem 2.1 of [7] also shows that every measurable symmetric and $[0,1]$-valued function $K$ arises as the limit of a convergent graph sequence.

The result of Theorem 2.1 from [7] proves existence and uniqueness of the limit graphon but it is not a constructive result. In fact, there is a natural "limit object" in the form of a symmetric measurable function $K: \Omega^{2} \rightarrow[0,1]$ which arises as a limit of an appropriate graph sequence but this limit is not explicitly known for every graph sequence. The natural question is then whether, given a graphon $K$, one can construct a sequence fo graphs $\left\{G_{n}\right\}_{n \in \mathbb{N}^{*}}$ whose limit is $K$. It turns out that there is such a random construction as we show in the next two sections.

\subsection{Random graphs}

The theory of random graphs was founded in the 50s-60s by Erdös and Rényi [12], who started the systematic study of the space of graphs with $n$ labeled vertices and $M=M(n)$ edges, with all graphs equiprobable. Nearly the same time, Gilbert [14] introduced the closely related model of random graphs on $n$ labeled vertices obtained as follows: join each pair $(i, j) \in[n]^{2}$ of vertices independently, with probability $p=p(n)$. These graphs are now known as Erdös-Rényi random graphs.

The aim is to turn the set of all graphs with $n$ vertices into a probability space. Intuitively we should be able to generate $G_{n}$ randomly as follows: for each edge $(i, j) \in[n]^{2}$, we decide by some random experiment wether or not $(i, j)$ shall be an edge of $G_{n}$, these experiments are performed independently.

Lovász et al. [7,22] defined a more general random graph model called $K$-random graph, as follows: given any symmetric measurable function $K: \Omega^{2} \rightarrow[0,1]$ and an integer $n \in \mathbb{N}^{*}$, generate $n$ independent numbers $\mathbf{X}_{1}, \ldots, \mathbf{X}_{n}$ from the uniform distribution on $\Omega$, and then connect nodes $i$ and $j$ with probability $K\left(\mathbf{X}_{i}, \mathbf{X}_{j}\right)$. The Erdös-Rényi graph corresponds to $K$ being the constant $p$-valued function. It was shown in Corollary 2.6 of [22] that this random construction provides a sequence of graphs $\left\{G_{n}\right\}_{n \in \mathbb{N}^{*}}$ which converges almost surely to the graphon $K$.

\section{3. $K$-random inhomogeneous graph model}

\subsubsection{Model generation}

The random graph models defined above are "homogeneous" in the sense that all vertices are exactly equivalent in the definition of the model. Furthermore, in a typical realization, most vertices are in some sense similar 
to most others. For example, the vertex degrees in the Erdös-Rényi model do not vary very much: their distribution is close to a Poisson distribution. However, many large real-world graphs are inhomogeneous. One reason is that the vertices may have been "born" at different times, with old and new vertices having very different properties. This has led to the introduction and analysis of many new random graph models designed to incorporate or explain inhomogeneous features. We here focus on a particular random graph model that will be used throughout. This random graph model is motivated by the construction of inhomogeneous random graphs proposed in $[3-5]$.

Definition 2.1. Fix $n \in \mathbb{N}^{*}$ and let $K: \Omega^{2} \rightarrow[0,1]$ be a symmetric measurable function. Generate the undirected graph $G_{n}=\left(V\left(G_{n}\right), E\left(G_{n}\right)\right) \stackrel{\text { def }}{=} G_{q_{n}}(n, K)$ as follows:

(1) Generate $n$ independent and identically distributed (i.i.d.) random variables $\left(\mathbf{X}_{1}, \ldots, \mathbf{X}_{n}\right) \stackrel{\text { def }}{=} \mathbf{X}$ from the uniform distribution on $\Omega$. Let $\left\{\mathbf{X}_{(i)}\right\}_{i=1}^{n}$ be the order statistics of the random vector $\mathbf{X}$, i.e. $\mathbf{X}_{(i)}$ is the $i$ th smallest value.

(2) Conditionally on $\mathbf{X}$, join each pair $(i, j) \in[n]^{2}$ of vertices independently, with probability $q_{n} \hat{K}_{n i j}^{\mathbf{X}}$, i.e. for every $(i, j) \in[n]^{2}, i \neq j$,

$$
\mathbb{P}\left((i, j) \in E\left(G_{n}\right) \mid \mathbf{X}\right)=q_{n} \hat{K}_{n i j}^{\mathbf{X}}
$$

where

$$
\stackrel{\wedge}{K}_{n i j}^{\mathbf{X}} \stackrel{\text { def }}{=} \min \left(\frac{1}{\left|\Omega_{n i j}^{\mathbf{X}}\right|} \int_{\Omega_{n i j}^{\mathbf{x}}} K(x, y) \mathrm{d} x \mathrm{~d} y, 1 / q_{n}\right),
$$

and

$$
\left.\left.\left.\left.\Omega_{n i j}^{\mathbf{X}} \stackrel{\text { def }}{=}\right] \mathbf{X}_{(i-1)}, \mathbf{X}_{(i)}\right] \times\right] \mathbf{X}_{(j-1)}, \mathbf{X}_{(j)}\right]
$$

where $q_{n}$ is non-negative and uniformly bounded in $n$.

A graph $G_{q_{n}}(n, K)$ generated according to this procedure is called a $K$-random inhomogeneous graph generated by a random sequence $\mathbf{X}$.

Following [3], we write the parameter $q_{n}$ as a subscript to emphasize that it is part of the normalization. For appropriate choices of $q_{n}$, this model allows to sample both dense and sparse graphs from the kernel $K$. In the latter, we think of a sparse graph generated from $K$, rather than a "sparse kernel" $q_{n} K$.

At this stage, the following important remark is in order.

Remark 2.2. In the context of numerical analysis, we are primarily interested not only in the error bounds of the discrete problem, but more importantly in the (nonasymptotic) rate of convergence. This is why our attention aims specifically at this graph model and not at the original inhomogeneous random model defined in $[3,5]$, i.e. the model constructed replacing $(2.3)$ by

$$
\mathbb{P}\left((i, j) \in E\left(G_{n}\right)\right)=\min \left(q_{n} K\left(\mathbf{X}_{i}, \mathbf{X}_{j}\right), 1\right)
$$

Our error bounds of the discrete problem $\left(\mathcal{P}_{n, \tau}^{d}\right)$ cover also this graph model, and more specifically, the first statements of Theorems 3.3 and 3.6 hold. However, with this model, even our convergence claim (not to mention the rate) of the discrete scheme does not hold unless the kernel $K$ and the initial data $g$ are additionally supposed almost everywhere continuous.

We denote by $\mathbf{x}=\left(\mathbf{x}_{1}, \ldots, \mathbf{x}_{n}\right)$ the realization of $\mathbf{X}$. To lighten the notation, we also denote

$$
\left.\left.\left.\left.\left.\left.\left.\left.\Omega_{n i}^{\mathbf{X}} \stackrel{\text { def }}{=}\right] \mathbf{X}_{(i-1)}, \mathbf{X}_{(i)}\right], \quad \Omega_{n i}^{\mathbf{x}} \stackrel{\text { def }}{=}\right] \mathbf{x}_{(i-1)}, \mathbf{x}_{(i)}\right], \quad \text { and } \quad \Omega_{n i j}^{\mathbf{x}} \stackrel{\text { def }}{=}\right] \mathbf{x}_{(i-1)}, \mathbf{x}_{(i)}\right] \times\right] \mathbf{x}_{(j-1)}, \mathbf{x}_{(j)}\right] \quad i, j \in[n] .
$$


As the realization of the random vector $\mathbf{X}$ is fixed, we define

$$
\stackrel{\wedge}{K}_{n i j}^{\mathbf{x}} \stackrel{\text { def }}{=} \min \left(\frac{1}{\left|\Omega_{n i j}^{\mathbf{x}}\right|} \int_{\Omega_{n i j}^{\mathbf{x}}} K(x, y) \mathrm{d} x \mathrm{~d} y, 1 / q_{n}\right), \quad \forall(i, j) \in[n]^{2}, \quad i \neq j .
$$

In the rest of the paper, the following random variables will be useful. Let $\lambda_{i j},(i, j) \in[n]^{2}, i \neq j$, be independent random variables such that $q_{n} \lambda_{i j}$ follows a Bernoulli distribution with parameter $q_{n} \hat{K}_{n i j}^{\mathbf{x}}$. We consider the independent random variables $\Upsilon_{i j}$ such that the distribution of $q_{n} \Upsilon_{i j}$ conditionally on $\mathbf{X}=\mathbf{x}$ is that of $q_{n} \lambda_{i j}$. Thus $q_{n} \Upsilon_{i j}$ follows a Bernoulli distribution with parameter $\mathbb{E}\left(q_{n} \stackrel{\hat{K}}{\mathbf{x}_{n i j}}\right)$, where $\mathbb{E}(\cdot)$ is the expectation operator (here with respect to the distribution of $\mathbf{X}$ ).

\subsubsection{Model assumptions}

We are now ready to formulate our assumptions on the graph sequence $\left\{G_{q_{n}}(n, K)\right\}_{n \in \mathbb{N}}$.

Assumption 2.3. We suppose that $q_{n}$ and $K$ are such that the following hold:

(A.1) $G_{q_{n}}(n, K)$ converges almost surely and its limit is the graphon $K$;

(A.2) $\sup _{n \geq 1} q_{n} \leq 1$.

At this stage, it is legitimate to discuss the validity of Assumption 2.3. As far as the boundedness assumption (A.2) is concerned, it is the least we can expect from $q_{n}$; otherwise the generated graphs are trivially empty. Of course, there is no loss of generality in taking 1 in the bound of (A.2). For (A.1), it is also reasonable as it allows one to assert that the randomly generated graph sequence has an (almost sure) limit object, which is the graphon $K$. The forthcoming proposition provides a large class of $K$-random graphs, corresponding to a particular scaling of $q_{n}$, for which both (A.1) and (A.2) hold. It is is inspired by the so-called non-uniform random graphs studied in Section 3.4 of [3]. This choice of $q_{n}$ allows to cover both dense and reasonably sparse graphs (see the discussion after Prop. 2.4) which are ubiquitous in various applications such as data (e.g. signal/image/point clouds) processing.

Proposition 2.4. Suppose $K: \Omega^{2} \rightarrow[0,1]$ is a symmetric measurable function. Choose the parameter $q_{n}=$ $n^{-g(n)}$ where $g(n)=o(1)$. Then, assumptions (A.1) and (A.2) are in force.

Proof. Since the graphon $K \in L^{\infty}\left(\Omega^{2}\right)$ and $q_{n}=n^{-o(1)}$, the arguments to prove ([3], Lems. 3.5 and 3.8), that were designed for the graph model described in Remark 2.2, can be adapted to cover our graph model with (2.3) to show that the sequence of random graphs $G_{q_{n}}(n, K)$ indeed converges almost surely to the graphon $K$ in the metric $d_{\text {sub }}$ (see [3], Sect. 2.1 for details about this metric). This shows (A.1). (A.2) is trivially verified.

The graph model of Proposition 2.4 encompasses the dense random graph model (i.e. with $\Theta\left(n^{2}\right)$ edges) extensively studied in [7,22], by taking the choice $g(n) \log (n)=C$, for $C>0$, and thus $q_{n}=e^{-C}$. This graph model allows also to generate sparse (but not too sparse) graphs. That is graphs with $o\left(n^{2}\right)$ but $\omega(n)$ edges, i.e. that the average degree tends to infinity with $n$. For example, one can take $g(n)=C \log (n)^{-\delta}$, where $\delta \in] 0,1\left[\right.$, in which case one has $q_{n}=\exp \left(-C \log (n)^{1-\delta}\right)=o(1)$, where such a choice of $q_{n}$ will control the level of density/sparsity.

\section{CONSISTENCY OF THE NONLOCAL $p$-LAPLACIAN ON RANDOM INHOMOGENEOUS GRAPHS}

Having defined the graph model, we are now in position to state our main error bounds between the discrete dynamics and their continuum counterparts. In Section 3.1, we first deal with the case where $\mathbf{X}$ is deterministic. Capitalizing on this result, we will then extend the bounds to the totally random model (i.e. where the nodes are also random) in Section 3.2 by a marginalization argument. 


\subsection{Networks on graphs generated by deterministic nodes}

We define the parameter $\delta(n)$ as the maximal size of the spacings between the ordered values $\mathbf{x}_{(i)}$

$$
\delta(n)=\max _{i \in[n]}\left|\mathbf{x}_{(i)}-\mathbf{x}_{(i-1)}\right| .
$$

Recall from $\left(\mathcal{P}_{n, \tau}^{d}\right)$ the definition of the time steps $\tau_{h}$ and maximal size $\tau$.

Next, we consider the following system of difference equations on $G_{q_{n}}(n, K)$ :

$$
\left\{\begin{array}{l}
\frac{u_{i}^{h}-u_{i}^{h-1}}{\tau_{h}}=\frac{1}{n} \sum_{j=1}^{n} \lambda_{i j}\left|u_{j}^{h-1}-u_{i}^{h-1}\right|^{p-2}\left(u_{j}^{h-1}-u_{i}^{h-1}\right), \quad(i, h) \in[n] \times[N], \\
u_{i}^{0}=g_{i}, i \in[n],
\end{array}\right.
$$

where $u_{i}^{h}$ is the value at vertex $i \in[n]$ and time $t_{h} \in[0, T]$, and

$$
g_{i}=\frac{1}{\left|\Omega_{n i}^{\mathbf{x}}\right|} \int_{\Omega_{n i}^{\mathbf{x}}} g(x) \mathrm{d} x .
$$

Recall from Section 2 that $\left\{q_{n} \lambda_{i j}\right\}_{i, j}$ are independent Bernoulli variables with parameters $\left\{q_{n} \hat{K}_{n i j}^{\mathbf{x}}\right\}_{i, j}$.

Before turning to our convergence result, we pause here to make the following observations.

Remark 3.1. Coming back to Definition 2.1, $G_{q_{n}}(n, K)$ is a random variable taking its values on the set of simple graphs. It is then important to keep in mind that the evolution equations we write involving random variables must be understood in this sense.

Remark 3.2. As the reader may have remarked, the sum in the right-hand side of $\left(\mathcal{P}_{n}^{d, d}\right)$ is divided by $n$ instead of a weighted sum with weights $\left|\mathbf{x}_{(i)}-\mathbf{x}_{(i-1)}\right|^{-1}$ which would be expected if we interpret this sum as a Riemann sum. The scaling by $n$ reminds us of an equidistant design regarding the space-discretization, despite the fact that the nodes are not necessarily equispaced. However, given that the $\mathbf{x}_{i}$ 's are realizations of i.i.d. uniform variables on $\Omega$, the uniform spacing choice still makes sense. Indeed, using classical results on order statistics of uniform variables, see, e.g. Section 1.7 of [27], it can be shown that each spacing $\mathbf{X}_{(i)}-\mathbf{X}_{(i-1)}$ concentrates around $1 / n$ for any $i \in[n]$.

We are now in position to tackle our main goal: comparing the solutions of the discrete and continuum problems and establish our rate of convergence. Since the two solutions do not live on the same spaces, it is reasonable to represent some intermediate model that is the continuum extension of the discrete problem, using the vector $U^{h}=\left(u_{1}^{h}, u_{2}^{h}, \ldots, u_{n}^{h}\right)^{\top}$ whose components solve the previous system $\left(\mathcal{P}_{n}^{d, d}\right)$ to obtain the following interpolation on $\Omega \times[0, T]$ (linear in time and piecewise constant in space)

$$
\left.\left.\check{u}_{n}(x, t)=\frac{t_{h}-t}{\tau_{h}} u_{i}^{h-1}+\frac{t-t_{h-1}}{\tau_{h}} u_{i}^{h} \quad \text { if } \quad x \in \Omega_{n i}^{\mathbf{x}}, \quad t \in\right] t_{h-1}, t_{h}\right],
$$

and a space-time piecewise constant approximation

$$
\bar{u}_{n}(x, t)=\sum_{i=1}^{n} \sum_{h=1}^{N} u_{i}^{h-1} \chi_{] t_{h-1}, t_{h}\right]}(t) \chi_{\Omega_{n i}^{\mathbf{x}}}(x) .
$$

Then, $\check{u}_{n}$ formally solves the following problem

$$
\begin{cases}\frac{\partial}{\partial t} \breve{u}_{n}(x, t)=-\Delta_{p}^{\Lambda_{n}}\left(\bar{u}_{n}(x, t)\right), & x \in \Omega, \quad t>0, \\ \check{u}_{n}(x, 0)=g_{n}(x), & x \in \Omega,\end{cases}
$$


where the random variable

$$
\Lambda_{n}(x, y)=\lambda_{i j} \quad \text { for } \quad(x, y) \in \Omega_{n i j}^{\mathbf{x}}
$$

and

$$
g_{n}(x)=g_{i} \quad \text { if } \quad x \in \Omega_{n i}^{\mathbf{x}}, i \in[n]
$$

Toward our goal of establishing error bounds, we need an intermediate discrete problem for the $p$-Laplacian. This is defined as

$$
\begin{cases}\frac{v_{i}^{h}-v_{i}^{h-1}}{\tau_{h}}=\frac{1}{n} \sum_{j=1}^{n} \hat{K}_{n i j}^{\mathrm{x}}\left|v_{j}^{h-1}-v_{i}^{h-1}\right|^{p-2}\left(v_{j}^{h-1}-v_{i}^{h-1}\right), & (i, h) \in[n] \times[N], \\ v_{i}^{0}=g_{i}, & i \in[n] .\end{cases}
$$

The discrete problem $\left(\hat{\mathcal{P}}_{n}^{d}\right)$ can also be viewed as a discrete $p$-Laplacian evolution problem over a weighted graph on $n$ vertices, where the weight of edge $(i, j)$ is $\hat{K}_{n i j}^{\mathbf{x}}$.

Using the vector $V^{h}=\left(v_{1}^{h}, v_{2}^{h}, \ldots, v_{n}^{h}\right)^{\top}$ whose components solve the system $\left(\hat{\mathcal{P}}_{n}^{d}\right)$, similarly to before, we define the following interpolation on $\Omega \times[0, T]$

$$
\left.\left.\check{v}_{n}(x, t)=\frac{t_{h}-t}{\tau_{h}} v_{i}^{h-1}+\frac{t-t_{h-1}}{\tau_{h}} v_{i}^{h} \quad \text { if } \quad x \in \Omega_{n i}^{\mathbf{x}}, \quad t \in\right] t_{h-1}, t_{h}\right],
$$

and a space-time piecewise constant interpolation

$$
\bar{v}_{n}(x, t)=\sum_{i=1}^{n} \sum_{h=1}^{N} v_{i}^{h-1} \chi_{] t_{h-1}, t_{h}\right]}(t) \chi_{\Omega_{n i}^{\mathrm{x}}}(x) .
$$

We also define the piecewise-constant extension $\stackrel{\wedge}{K}_{n}$ on $\Omega^{2}$

$$
\hat{K}_{n}(x, y)=\sum_{(i, j) \in[n]^{2}} \hat{K}_{n i j}^{\mathbf{x}} \chi_{\Omega_{n i j}^{\mathbf{x}}}(x, y)
$$

Then, by construction, $\check{v}_{n}(x, t)$ formally solves the following problem

$$
\begin{cases}\frac{\partial}{\partial t} \breve{v}_{n}(x, t)=-\boldsymbol{\Delta}_{p}^{\hat{K}_{n}}\left(\bar{v}_{n}(x, t)\right), & x \in \Omega, t>0, \\ \check{v}_{n}(x, 0)=g_{n}(x), & x \in \Omega\end{cases}
$$

where

$$
g_{n}(x)=g_{i} \quad \text { for } \quad x \in \Omega_{n i}^{\mathbf{x}}, i \in[n] .
$$

The first main result of the paper is the following theorem. 
Theorem 3.3. Suppose that $p \in] 1,+\infty\left[, K \in L^{\infty}\left(\Omega^{2}\right)\right.$ is a symmetric and measurable mapping, and $g \in$ $L^{\infty}(\Omega)$. Let $u$ and $U^{h}$ denote the solutions to $(\mathcal{P})$ and $\left(\mathcal{P}_{n}^{d, d}\right)$, respectively. Let $\check{u}_{n}$ be the continuum extension of $U^{h}$ given in (3.2). Then, the following hold:

(i) for $T>0$, there exist positive constants $C_{1}$ and $C_{2}$, independent of $n$ and $T$, such that for any $\beta>0$

$$
\begin{aligned}
\left\|u-\check{u}_{n}\right\|_{C\left(0, T ; L^{p}(\Omega)\right)} \leq & C_{1} T \exp (O(T))\left(\left(\beta \frac{\log (n)}{n}+\frac{\max \left(q_{n}^{-(p-1)}, q_{n}^{-p / 2}\right)}{n^{p / 2}}\right)^{1 / p}\right. \\
& \left.+\left\|K-\hat{K}_{n}\right\|_{L^{p}\left(\Omega^{2}\right)}+\left\|g-g_{n}\right\|_{L^{p}(\Omega)}+\tau\right)
\end{aligned}
$$

with probability at least $1-n^{-C_{2} q_{n}^{2 p-1} \beta}$.

(ii) Suppose furthermore that $g \in \operatorname{Lip}\left(s, L^{q}(\Omega)\right)$ and $\left.\left.K \in \operatorname{Lip}\left(s^{\prime}, L^{q}\left(\Omega^{2}\right)\right), q \in[1,+\infty], s, s^{\prime} \in\right] 0,1\right]$, and $q_{n}\|K\|_{L^{\infty}\left(\Omega^{2}\right)} \leq 1$. Then, for $T>0$, there exist positive constants $C_{1}$ and $C_{2}$, independent of $n$ and $T$, such that for any $\beta>0$

$$
\begin{aligned}
\left\|u-\check{u}_{n}\right\|_{C\left(0, T ; L^{p}(\Omega)\right)} \leq & C_{1} T \exp (O(T))\left(\left(\beta \frac{\log (n)}{n}+\frac{\max \left(q_{n}^{-(p-1)}, q_{n}^{-p / 2}\right)}{n^{p / 2}}\right)^{1 / p}\right. \\
& \left.+\delta(n)^{\min \left(s, s^{\prime}\right) \min (1, q / p)}+\tau\right)
\end{aligned}
$$

with probability at least $1-n^{-C_{2} q_{n}^{2 p-1} \beta}$, where $\delta(n)$ is the spacing parameter defined in (3.1).

Before proceeding to the proof, some remarks are in order.

\section{Remark 3.4.}

(i) The constant $C_{1}$ in (3.7) depends on $p$ and the data via $\|g\|_{L^{\infty}(\Omega)}$ and $\|K\|_{L^{\infty}\left(\Omega^{2}\right)}$. For the bound (3.8), it also depends on $\left(q, s, s^{\prime}\right)$. Similarly, the constant in the $O(T)$ term in the exponential can be made explicit and depends on $p,\|g\|_{L^{\infty}(\Omega)}$ and $\|K\|_{L^{\infty}\left(\Omega^{2}\right)}$.

(ii) Thanks to the well-known inequality

$$
\left.\left.(a+b)^{\alpha} \leq a^{\alpha}+b^{\alpha}, \quad \forall \alpha \in\right] 0,1\right] \text { and } a, b \geq 0,
$$

it is clear that the first term in the bounds (3.7) and (3.8) can be replaced by

$$
\beta^{1 / p}\left(\frac{\log (n)}{n}\right)^{1 / p}+\frac{\max \left(q_{n}^{-(1-1 / p)}, q_{n}^{-1 / 2}\right)}{n^{1 / 2}} .
$$

(iii) The last term in the latter bound can be rewritten as

$$
n^{-1 / 2} \max \left(q_{n}^{-(1-1 / p)}, q_{n}^{-1 / 2}\right)= \begin{cases}\left(q_{n} n\right)^{-1 / 2} & \text { if } p \in] 1,2], \\ q_{n}^{1 / p}\left(q_{n}^{2} n\right)^{-1 / 2} & \text { if } p>2 .\end{cases}
$$

Thus, if $\inf _{n \geq 1} q_{n}>0$, as is the case when the graph is dense (see discussion after Prop. 2.4), then the term (3.10) is in the order of $n^{-1 / 2}$ with probability at least $1-n^{-c \beta}$ for some $c>0$. If $q_{n}$ is allowed to be $o(1)$, i.e. sparse graphs (see Prop. 2.4), then (3.10) is $o(1)$ if either $q_{n} n \rightarrow+\infty$ for $\left.\left.p \in\right] 1,2\right]$, or $q_{n}^{2} n \rightarrow+\infty$ for $p>2$. The probability of success is at least $1-e^{-C_{2} \beta \log (n)^{1-\delta}}$ provided that $q_{n}=\log (n)^{-\delta /(2 p-1)}$, with $\delta \in\left[0,1\left[\right.\right.$. Observe that all these conditions on $q_{n}$ are fulfilled by the graph model of Proposition 2.4 for $g(n)=\delta /(2 p-1) \log (\log (n)) / \log (n)$. 
(iv) In fact, if $\inf _{n \geq 1} q_{n} \geq c>0$, then we have $\sum_{n \geq 1} n^{-C_{2} q_{n}^{2 p-1} \beta} \leq \sum_{n \geq 1} n^{-C_{2} c^{2 p-1} \beta}<+\infty$ provided that $\beta>\left(C_{2} c^{2 p-1}\right)^{-1}$. Thus, if this holds, invoking the (first) Borel-Cantelli lemma, it follows that the bounds of Theorem 3.3 hold almost surely. The same reasoning carries over for the bounds of Theorem 3.6.

(v) For finite fixed $T$, the term $T \exp \left(c_{1} T\right)$, for $c_{1}>0$, in the bound becomes a constant. One can even allow for time intervals of size $T=c_{2} \log (n), c_{2}>0$, in which case this term scales as $O\left(n^{c_{1} c_{2}} \log (n)\right)$. Thus this term can be dominated by the other rates in $n$ if $c_{1} c_{2}$ is sufficiently small (see Rem. 3.7(ii) for details).

(vi) One may wonder if the functional space assumption made on $g$ and $K$ in claim (ii) is reasonable or even makes sense. The answer is affirmative. Indeed, Lipschitz spaces are rich enough to include both functions with discontinuities and even fractal structure. For instance, from [21], one can show that the graphon corresponding to the nearest neighbour graphs, which are very popular in practice (e.g. in image processing $[10,11]$ ), are typical examples satisfying assumptions (A.1) and (A.2) with $q_{n}=1$ and $K$ is a $\{0,1\}$-valued function living on the space of bounded variation functions, which in turn is $\operatorname{Lip}\left(1, L^{1}\left(\Omega^{2}\right)\right)$.

To prove Theorem 3.3, we first show the following key lemma.

Lemma 3.5. Under the assumptions of Theorem 3.3, for $T>0$, there exist positive constants $C_{1}$ and $C_{2}$, independent of $n$ and $T$, such that for any $\beta>0$

$$
\mathbb{P}\left(\left\|\check{v}_{n}-\check{u}_{n}\right\|_{C\left(0, T ; L^{p}(\Omega)\right)} \geq \varepsilon\right) \leq n^{-C_{2} q_{n}^{2 p-1} \beta},
$$

where

$$
\varepsilon=C_{1} T \exp (O(T))\left(\left(\beta \frac{\log (n)}{n}+\max \left(q_{n}^{-(p-1)}, q_{n}^{-p / 2}\right) \frac{1}{n^{p / 2}}\right)^{1 / p}+\tau\right) .
$$

Proof of Lemma 3.5. For $1<p<+\infty$, we define the function

$$
\begin{aligned}
& \Psi: \mathbb{R} \rightarrow \mathbb{R} \\
& x \mapsto|x|^{p-2} x=\operatorname{sign}(x)|x|^{p-1} .
\end{aligned}
$$

First, for an appropriate choice of $\tau_{h}$, using Lemma 5.1 of [17], we have that both $\left(\mathcal{P}_{n}^{d, d}\right)$ and $\left(\hat{\mathcal{P}}_{n}^{d}\right)$ are well posed. In turn $U^{h}$ and $V^{h}$ are bounded and $V^{h}$ uniquely solves $\left(\hat{\mathcal{P}}_{n}^{d}\right)$, and similarly for $\check{u}_{n}$ and $\check{v}_{n}$ as solutions to $\left(\mathcal{P}_{n}\right)$ and $\left(\hat{\mathcal{P}}_{n}\right)$. Observe also that $\check{v}_{n}(\cdot, t)$ and $\check{u}_{n}(\cdot, t)$ are both constants over $\Omega_{n i}^{\mathbf{x}}$. Similarly, $\bar{v}_{n}(\cdot, t)$ and $\bar{u}_{n}(\cdot, t)$ are also constants over the cell $\Omega_{n i}^{\mathbf{x}}$. We therefore used the shorthand notations for the vector-valued functions $\overline{\mathbf{u}}_{n}(t)=\left(\overline{\mathbf{u}}_{n i}(t)\right)_{i \in[n]} \stackrel{\text { def }}{=}\left(\bar{u}_{n}\left(\mathbf{x}_{i}, t\right)\right)_{i \in[n]}$ and $\overline{\mathbf{v}}_{n}(t)=\left(\overline{\mathbf{v}}_{n}(t)\right)_{i \in[n]} \stackrel{\text { def }}{=}\left(\bar{v}_{n}\left(\mathbf{x}_{i}, t\right)\right)_{i \in[n]}$, and likewise for $\check{\mathbf{u}}_{n}(t)$ and $\check{\mathbf{v}}_{n}(t)$. Let us denote $\check{\xi}_{n}(t)=\check{\mathbf{u}}_{n}(t)-\check{\mathbf{v}}_{n}(t)$ and $\bar{\xi}_{n}(t)=\overline{\mathbf{u}}_{n}(t)-\overline{\mathbf{v}}_{n}(t)$. By subtracting both sides of $\left(\mathcal{P}_{n}\right)$ from those of $\left(\hat{\mathcal{P}}_{n}\right)$, evaluated at the cell $\Omega_{n i}^{\mathbf{x}}$, we obtain

$$
\begin{aligned}
\frac{\mathrm{d}}{\mathrm{d} t} \check{\xi}_{n i}(t) & =\frac{1}{n} \sum_{j=1}^{n}\left(\lambda_{i j} \Psi\left(\overline{\mathbf{u}}_{n j}(t)-\overline{\mathbf{u}}_{n i}(t)\right)-\hat{K}_{n i j}^{\mathbf{x}} \Psi\left(\overline{\mathbf{v}}_{n j}(t)-\overline{\mathbf{v}}_{n i}(t)\right)\right) \\
& =Z_{n i}(t)+\frac{1}{n} \sum_{j=1}^{n} \hat{K}_{n i j}^{\mathbf{x}}\left(\Psi\left(\overline{\mathbf{u}}_{n j}(t)-\overline{\mathbf{u}}_{n i}(t)\right)-\Psi\left(\overline{\mathbf{v}}_{n j}(t)-\overline{\mathbf{v}}_{n i}(t)\right)\right)
\end{aligned}
$$

where

$$
Z_{n i}(t)=\frac{1}{n} \sum_{j=1}^{n}\left(\lambda_{i j}-\hat{K}_{n i j}^{\mathbf{x}}\right) \alpha_{i j}(t) \text { and } \alpha_{i j}(t)=\Psi\left(\overline{\mathbf{u}}_{n j}(t)-\overline{\mathbf{u}}_{n i}(t)\right), \forall(i, j) \in[n]^{2}, t \in[0, T]
$$


By our discussion above, we have $\sup _{(i, j) \in[n]^{2}, t \in[0, T]}\left|\alpha_{i j}(t)\right|<+\infty$. We multiply both sides of (3.11) by $\frac{1}{n} \Psi\left(\check{\xi}_{n i}(t)\right)$ and sum over $i$ to obtain

$$
\frac{1}{p} \frac{\mathrm{d}}{\mathrm{d} t}\left\|\check{\xi}_{n}(t)\right\|_{p, n}^{p}=\frac{1}{n} \sum_{i=1}^{n} Z_{n i}(t) \Psi\left(\check{\xi}_{n i}(t)\right)+\frac{1}{n^{2}} \sum_{i, j=1}^{n} \hat{K}_{n i j}^{\mathbf{x}}\left(\Psi\left(\overline{\mathbf{u}}_{n j}(t)-\overline{\mathbf{u}}_{n i}(t)\right)-\Psi\left(\overline{\mathbf{v}}_{n j}(t)-\overline{\mathbf{v}}_{n i}(t)\right)\right) \Psi\left(\check{\xi}_{n i}(t)\right) .
$$

We estimate the first term on the right-hand side of (3.13) using the Hölder inequality, to get

$$
\frac{1}{n}\left|\sum_{i=1}^{n} Z_{n i}(t) \Psi\left(\check{\xi}_{n i}(t)\right)\right| \leq \frac{1}{n}\left(\sum_{i=1}^{n}\left|Z_{n i}(t)\right|^{p}\right)^{\frac{1}{p}} \times\left(\sum_{i=1}^{n}\left|\check{\xi}_{n i}(t)\right|^{p}\right)^{\frac{p-1}{p}} \leq\left\|Z_{n}(t)\right\|\left\|_{p, n}\right\| \check{\xi}_{n}(t) \|_{p, n}^{p-1} .
$$

Now, using the fact that $\hat{K}_{n i j}^{\mathbf{x}} \leq\|K\|_{L^{\infty}\left(\Omega^{2}\right)}$ (see $\left.(2.4)\right), \forall(i, j) \in[n]^{2}$, and applying a generalized mean value theorem ([17], Cor. B.1) to the function $\Psi$, since $p>1$, between $a_{i j}(t)=\overline{\mathbf{v}}_{n j}(t)-\overline{\mathbf{v}}_{n i}(t)$ and $b_{i j}(t)=$ $\overline{\mathbf{u}}_{n j}(t)-\overline{\mathbf{u}}_{n i}(t)$ (without loss of generality, we suppose that $b_{n i j}(t)>a_{i j}(t)$ ), we get

$$
\begin{array}{r}
\mid \frac{1}{n^{2}} \sum_{i, j=1}^{n} \hat{K}_{n i j}^{\mathbf{x}}\left(\Psi\left(\overline{\mathbf{u}}_{n j}(t)-\overline{\mathbf{u}}_{n i}(t)\right)-\Psi\left(\overline{\mathbf{v}}_{n j}(t)-\overline{\mathbf{v}}_{n i}(t)\right) \Psi\left(\xi_{n i}(t)\right) \mid\right. \\
\leq \frac{(p-1)\|K\|_{L^{\infty}\left(\Omega^{2}\right)}}{n^{2}} \sum_{i, j=1}^{n}\left|\bar{\xi}_{n j}-\bar{\xi}_{n i}\right|\left|\eta_{i j}(t)\right|^{p-2}\left|\check{\xi}_{n i}\right|^{p-1}
\end{array}
$$

where $\eta_{i j}(t)$ is an intermediate value between $a_{i j}(t)$ and $b_{i j}(t)$. Using that fact that $g \in L^{\infty}(\Omega)$ and the construction of $\overline{\mathbf{u}}_{n}(\cdot)$, we deduce from Theorem 3.1(ii) of [17] that for any $(i, j) \in[n]^{2}$ and $t \in[0, T]$, we have for $p \geq 2$

$$
\left|\eta_{i j}(t)\right|^{p-2} \leq\left|\overline{\mathbf{u}}_{n j}(t)-\overline{\mathbf{u}}_{n i}(t)\right|^{p-2} \leq\left(2\|u(\cdot, t)\|_{L^{\infty}(\Omega)}\right)^{p-2} \leq\left(2\|g\|_{L^{\infty}(\Omega)}\right)^{p-2} .
$$

For $p \in] 1,2\left[\right.$, since $\inf _{(i, j, t) \in[n]^{2} \times[0, T]}\left|\eta_{i j}(t)\right|=C^{\prime}>0$, we have

$$
\left|\eta_{i j}(t)\right|^{p-2} \leq C^{\prime p-2}<+\infty .
$$

Altogether, we obtain

$$
\left|\eta_{i j}(t)\right|^{p-2} \leq \max \left(\left(2\|g\|_{L^{\infty}(\Omega)}\right)^{p-2}, C^{\prime p-2}\right) .
$$

Let $C_{2}=\max \left(\left(2\|g\|_{L^{\infty}(\Omega)}\right)^{p-2}, C^{\prime p-2}\right)\|K\|_{L^{\infty}\left(\Omega^{2}\right)}$. Inserting (3.16) into (3.15), and then using the Hölder and triangle inequalities, it follows that

$$
\begin{aligned}
\mid \frac{1}{n^{2}} \sum_{i, j=1}^{n} & \hat{K}_{n i j}^{\mathbf{x}}\left(\Psi\left(\overline{\mathbf{u}}_{n j}(t)-\overline{\mathbf{u}}_{n i}(t)\right)-\Psi\left(\overline{\mathbf{v}}_{n j}(t)-\overline{\mathbf{v}}_{n i}(t)\right) \Psi\left(\check{\xi}_{n i}(t)\right) \mid\right. \\
& \leq C_{2} \frac{p-1}{n^{2}} \sum_{i, j=1}^{n}\left|\bar{\xi}_{n j}(t)-\bar{\xi}_{n i}(t)\right|\left|\check{\xi}_{n i}\right|^{p-1} \\
& \leq C_{2} \frac{p-1}{n^{2}}\left(\left(\sum_{i, j=1}^{n}\left|\bar{\xi}_{n j}(t)-\bar{\xi}_{n i}(t)\right|^{p}\right)^{\frac{1}{p}}\left(\sum_{i, j}\left|\check{\xi}_{n i}(t)\right|^{p}\right)^{\frac{p-1}{p}}\right)
\end{aligned}
$$




$$
\begin{aligned}
& \leq C_{2} \frac{p-1}{n^{2}}\left(\left(\sum_{i, j=1}^{n}\left|\bar{\xi}_{n j}(t)\right|^{p}\right)^{\frac{1}{p}}+\left(\sum_{i, j=1}^{n}\left|\bar{\xi}_{n i}(t)\right|^{p}\right)^{\frac{1}{p}}\right)\left(n^{\frac{2(p-1)}{p}}\left(\frac{1}{n} \sum_{i=1}^{n}\left|\check{\xi}_{n i}(t)\right|^{p}\right)^{\frac{p-1}{p}}\right) \\
& \leq C_{2} \frac{p-1}{n^{2}}\left(2 n^{\frac{2}{p}}\left\|\bar{\xi}_{n}(t)\right\|_{p, n}\right)\left(n^{\frac{2(p-1)}{p}}\left\|\check{\xi}_{n}(t)\right\|_{p, n}^{p-1}\right) \\
& \leq 2 C_{2}(p-1)\left\|\bar{\xi}_{n}(t)\right\|_{p, n}\left\|\check{\xi}_{n}(t)\right\|_{p, n}^{p-1} .
\end{aligned}
$$

Using the triangle inequality combined with Lemma 5.2 of [17], we have

$$
\begin{aligned}
\left\|\bar{\xi}_{n}(t)\right\|_{p, n} & =\left\|\overline{\mathbf{v}}_{n}(t)-\overline{\mathbf{u}}_{n}(t)\right\|_{p, n} \\
& \leq\left\|\overline{\mathbf{v}}_{n}(t)-\check{\mathbf{v}}_{n}(t)\right\|_{p, n}+\left\|\check{\mathbf{v}}_{n}(t)-\check{\mathbf{u}}_{n}(t)\right\|_{p, n}+\left\|\check{\mathbf{u}}_{n}(t)-\overline{\mathbf{u}}_{n}(t)\right\|_{p, n} \\
& \leq C \tau+\left\|\check{\xi}_{n}(t)\right\|_{p, n}+C^{\prime} \tau \\
& \leq C^{\prime \prime} \tau+\left\|\check{\xi}_{n}(t)\right\|_{p, n}
\end{aligned}
$$

Putting together (3.13), (3.14), (3.17) and (3.18), we have

$$
\begin{aligned}
\frac{\mathrm{d}}{\mathrm{d} t}\left\|\check{\xi}_{n}(t)\right\|_{p, n}^{p} & \leq\left\|Z_{n}(t)\right\|_{p, n}\left\|\check{\xi}_{n}(t)\right\|_{p, n}^{p-1}+2 C_{2}(p-1)\left(C^{\prime \prime} \tau+\left\|\check{\xi}_{n}(t)\right\|_{p, n}\right)\left\|\check{\xi}_{n}(t)\right\|_{p, n}^{p-1} \\
& \leq\left(2 C_{3}(p-1) \tau+\left\|Z_{n}(t)\right\|_{p, n}\right)\left\|\check{\xi}_{n}(t)\right\|_{p, n}^{p-1}+2 C_{2}(p-1)\left\|\check{\xi}_{n}(t)\right\|_{p, n}^{p} .
\end{aligned}
$$

Then, from (3.19) via the Gronwall's inequality in its differential form (see, e.g. [13], Appendix B), we obtain

$$
\left\|\check{u}_{n}-\check{v}_{n}\right\|_{C\left(0, T ; L^{p}(\Omega)\right)}=\sup _{t \in[0, T]}\left\|\check{\xi}_{n}(t)\right\|_{p, n} \leq\left(2 C_{3} T \tau+\int_{0}^{T}\left\|Z_{n}(t)\right\|_{p, n} \mathrm{~d} t\right) \exp \left(2 C_{2} T\right) .
$$

It remains to bound $\int_{0}^{T}\left\|Z_{n}(t)\right\|_{p, n} \mathrm{~d} t$. For this purpose, we use Lemma A.1 (see Sect. A.1) ${ }^{7}$. Thus, plugging the bound of Lemma A.1(i) into inequality (3.20), we get the desired conclusion.

We are now ready to prove our main result.

Proof of Theorem 3.3. (i) Using the triangle inequality, we have

$$
\left\|u-\check{u}_{n}\right\|_{C\left(0, T ; L^{p}(\Omega)\right)} \leq\left\|u-\check{v}_{n}\right\|_{C\left(0, T ; L^{p}(\Omega)\right)}+\left\|\check{v}_{n}-\check{u}_{n}\right\|_{C\left(0, T ; L^{p}(\Omega)\right)} .
$$

Since by construction $\hat{K}_{n}$ is a bounded mapping, we bound the first term on the right-hand side of (3.21) using Theorem 5.1 of $[17]^{8}$ to get

$$
\left\|u-\check{v}_{n}\right\|_{C\left(0, T ; L^{p}(\Omega)\right)}=O\left(T \exp (O(T))\left(\left\|K-\hat{K}_{n}\right\|_{L^{p}\left(\Omega^{2}\right)}+\left\|g-g_{n}\right\|_{L^{p}(\Omega)}+\tau\right)\right),
$$

Claim (3.7) then follows by plugging (3.22) and Lemma 3.5 into (3.21).

(ii) Our assumption on $q_{n}$ together with (2.7) and (3.6) entail that

$$
\hat{K}_{n}(x, y)=\sum_{(i, j) \in[n]^{2}} K_{n i j} \chi_{\Omega_{n i j}^{\mathbf{x}}}(x, y), \quad K_{n i j}=\frac{1}{\left|\Omega_{n i j}^{\mathbf{X}}\right|} \int_{\Omega_{n i j}^{\mathbf{x}}} K(x, y) \mathrm{d} x \mathrm{~d} y .
$$

\footnotetext{
${ }^{7}$ This inequality is sharp as can be seen for instance from assertion (ii) of Lemma A.1, at least for $p \geq 2$.

${ }^{8}$ Here, we have made the constant explicit in $T$ compared to the statement in Theorem 5.1 of [17].
} 
Since $g \in \operatorname{Lip}\left(s, L^{q}(\Omega)\right)$ and $K \in \operatorname{Lip}\left(s^{\prime}, L^{q}\left(\Omega^{2}\right)\right)$, we can invoke Lemma A.4 to get

$$
\left\|K-\hat{K}_{n}\right\|_{L^{p}\left(\Omega^{2}\right)} \leq C\left(p, q, s^{\prime}\right) \delta(n)^{s^{\prime} \min (1, q / p)} \text { and }\left\|g-g_{n}\right\|_{L^{p}(\Omega)} \leq C(p, q, s) \delta(n)^{s \min (1, q / p)} .
$$

Inserting the bound (3.23) into (3.7), and using the fact that $\delta(n)<1$, yields (3.8).

\subsection{Networks on graphs generated by random nodes}

Let us now turn to the totally random graph model. Consider the following system of difference equations on the totally random graph $G_{q_{n}}(n, K)^{9}$ :

$$
\left\{\begin{array}{l}
\frac{u_{i}^{h}-u_{i}^{h-1}}{\tau_{h}}=\frac{1}{n} \sum_{\left\{j:(i, j) \in E\left(G_{q_{n}}(n, K)\right)\right\}}\left|u_{j}^{h-1}-u_{i}^{h-1}\right|^{p-2}\left(u_{j}^{h-1}-u_{i}^{h-1}\right), \quad h \in[N] \\
u_{i}^{0}=g_{i}, i \in[n] .
\end{array}\right.
$$

As we have done before, we consider the continuum extension of the solution vector $U^{h}=\left(u_{1}^{h}\right.$, $\left.u_{2}^{h}, \ldots, u_{n}^{h}\right)^{\top}$, that is a linear interpolation on $\Omega \times[0, T]$

$$
\left.\left.\check{u}_{n}(x, t)=\frac{t_{h}-t}{\tau_{h}} u_{i}^{h-1}+\frac{t-t_{h-1}}{\tau_{h}} u_{i}^{h} \quad \text { if } \quad x \in \Omega_{n i}^{\mathbf{X}}, \quad t \in\right] t_{h-1}, t_{h}\right],
$$

and a piecewise approximation

$$
\bar{u}_{n}(x, t)=\sum_{i=1}^{n} \sum_{h=1}^{N} u_{i}^{h-1} \chi_{] t_{h-1}, t_{h}\right]}(t) \chi_{\Omega_{n i}^{\mathbf{x}}}(x)
$$

Then, we have

$$
\begin{cases}\frac{\partial}{\partial t} \check{u}_{n}(x, t)=-\Delta_{p}^{\Gamma_{n}}\left(\bar{u}_{n}(x, t)\right), & x \in \Omega, t>0 \\ \check{u}_{n}(x, 0)=g_{n}(x), & x \in \Omega\end{cases}
$$

where

$$
g_{n}(x)=g_{i} \quad \text { if } \quad x \in \Omega_{n i}^{\mathbf{X}}, i \in[n]
$$

and the random variable $\Gamma_{n}$ is such that

$$
\Gamma_{n}(x, y)=\Upsilon_{i j} \quad \text { for } \quad(x, y) \in \Omega_{n i j}^{\mathbf{X}}
$$

If conditioned with respect to a realization $\mathbf{x}=\left(\mathbf{x}_{1}, \ldots, \mathbf{x}_{n}\right)$ of the random vector $\mathbf{X}$, problem $\left(\mathcal{P}_{n}^{r, d}\right)$ can be rewritten on $G_{q_{n}}(n, K)$ in the following form

$$
\begin{cases}\frac{u_{i}^{h}-u_{i}^{h-1}}{\tau_{h}}=\frac{1}{n} \sum_{j=1}^{n} \lambda_{i j}\left|u_{j}^{h-1}-u_{i}^{h-1}\right|^{p-2}\left(u_{j}^{h-1}-u_{i}^{h-1}\right), & (i, h) \in[n] \times[N], \\ u_{i}^{0}=g_{i}, & i \in[n] .\end{cases}
$$

By capitalizing on the results obtained for the the case where $\left\{G_{q_{n}}(n, K)\right\}_{n \in \mathbb{N}}$ was generated by the deterministic sequence $\mathbf{x}$, we get the following result.

\footnotetext{
${ }^{9}$ Recall again from Remark 3.1, that rigorously speaking, each variable involved in the problems and equations of this section should be understood as random.
} 
Theorem 3.6. Suppose that $p \in] 1,+\infty\left[, K \in L^{\infty}\left(\Omega^{2}\right)\right.$ is a symmetric and measurable mapping, and $g \in$ $L^{\infty}(\Omega)$. Let $u$ and $U_{h}$ denote the solutions to $(\mathcal{P})$ and $\left(\mathcal{P}_{n}^{r, d}\right)$, respectively. Let $\check{u}_{n}$ be the continuum extension of $U_{h}$ given in (3.24). Then, the following hold:

(i) For $T>0$, there exist positive constants $C_{1}$ and $C_{2}$, independent of $n$ and $T$, such that for any $\beta>0$

$$
\begin{aligned}
\left\|u-\check{u}_{n}\right\|_{C\left(0, T ; L^{p}(\Omega)\right)} \leq & C_{1} T \exp (O(T))\left(\left(\beta \frac{\log (n)}{n}+\frac{\max \left(q_{n}^{-(p-1)}, q_{n}^{-p / 2}\right)}{n^{p / 2}}\right)^{1 / p}\right. \\
& \left.+\left\|K-\hat{K}_{n}\right\|_{L^{p}\left(\Omega^{2}\right)}+\left\|g-g_{n}\right\|_{L^{p}(\Omega)}+\tau\right)
\end{aligned}
$$

with probability at least $1-n^{-C_{2} q_{n}^{2 p-1} \beta}$.

(ii) Suppose furthermore that $g \in \operatorname{Lip}\left(s, L^{q}(\Omega)\right)$ and $\left.\left.K \in \operatorname{Lip}\left(s^{\prime}, L^{q}\left(\Omega^{2}\right)\right), s, s^{\prime} \in\right] 0,1\right]$, and $q_{n}\|K\|_{L^{\infty}\left(\Omega^{2}\right)} \leq 1$.

Let $\theta \stackrel{\text { def }}{=} \min \left(s, s^{\prime}\right) \min (1, q / p)$. Then, for $T>0$, there exist positive constants $C_{1}$ and $C_{2}$, independent of $n$ and $T$, such that for any $\beta>0$ and $t \in] 0, e[$

$$
\left\|u-\check{u}_{n}\right\|_{C\left(0, T ; L^{p}(\Omega)\right)} \leq C_{1} T \exp (O(T))\left(\left(\beta \frac{\log (n)}{n}+\frac{\max \left(q_{n}^{-(p-1)}, q_{n}^{-p / 2}\right)^{1 / p}}{n^{p / 2}}\right)+\left(\frac{t \log (n)}{n}\right)^{\theta}+\tau\right),
$$

with probability at least $1-\left(n^{-C_{2} q_{n}^{2 p-1} \beta}+n^{-t}\right)$.

\section{Remark 3.7.}

(i) The dependence of the constant $C$ in the parameters is similar to Remark 3.4(i).

(ii) As observed in Remark 3.4(v), one can take $T=c_{2} \log (n)$, in which case $T \exp \left(c_{1} T\right)=c_{2} n^{c_{1} c_{2}} \log (n)$, with $c_{1}, c_{2}>0$. Consequently, if one sets $q_{n}=\log (n)^{-\delta /(2 p-1)}$, for $\left.\delta \in\right] 0,1[$ (see Rem. 3.4(iii)), then the bound in $(3.27)$ scales as $O\left(\frac{\log (n)^{s}}{n^{\min (1 / p, 1 / 2, \theta)-c_{1} c_{2}}}\right)$, for some $s>0$, which converges to 0 provided that $c_{1} c_{2}<\min (1 / p, 1 / 2, \theta)$.

As a preparatory step to prove Theorem 3.6, the following lemma is instrumental. It establishes that the spacings between the $n$ uniformly distributed nodes are $O(\log (n) / n)$ with high probability.

Lemma 3.8. Consider the sequence of random spacings $\left(\mathbf{X}_{(1)}, \mathbf{X}_{(2)}-\mathbf{X}_{(1)}, \ldots, 1-\mathbf{X}_{(n)}\right)$, where we recall $\left\{\mathbf{X}_{(i)}\right\}_{i=1}^{n}$ are the order statistics of $\mathbf{X}$. Let $\left.t \in\right] 0, e[$. Then, for any $i \in[n]$

$$
\delta_{i} \stackrel{\text { def }}{=} \mathbf{X}_{(i)}-\mathbf{X}_{(i-1)} \leq t \frac{\log (n)}{n}
$$

with probability at least $1-n^{-t}$.

Proof of Lemma 3.8. Since $\mathbf{X}_{i}$ are i.i.d. uniform random variables on $\Omega$, we have, by virtue of Theorem 1.6.7 from [27] that the random variables $\delta_{i}, i \in[n]$, have the same distribution as the random variables $Z_{i} / \sum_{k=1}^{n+1} Z_{k}$, where $Z_{1}, \ldots, Z_{n+1}$ are i.i.d standard exponential random variables. In addition, invoking ([27], Lem. 1.6.6), we know that $S_{n+1} \stackrel{\text { def }}{=} \sum_{k=1}^{n+1} Z_{k}$ is a Gamma random variable with parameters $(1, n+1)$ (thus having the density $\left.f_{S_{n+1}}(s)=e^{-s} s^{n} / n !, s \geq 0\right)$. 
Now, combining these two observations, we obtain by straightforward integral calculations that for any $\varepsilon \in[0,1[$

$$
\begin{aligned}
\mathbb{P}\left(\delta_{i} \geq \varepsilon\right)=\mathbb{P}\left(Z_{i} \geq \varepsilon S_{n+1}\right) & =\mathbb{P}\left((1-\varepsilon) Z_{i} \geq \varepsilon\left(S_{n+1}-Z_{i}\right)\right) \\
& =\mathbb{P}\left(Z_{n+1} \geq \frac{\varepsilon}{1-\varepsilon} S_{n}\right) \\
& =\int_{0}^{+\infty} \mathbb{P}\left(Z_{n+1} \geq \frac{\varepsilon}{1-\varepsilon} s\right) f_{S_{n}}(s) \mathrm{d} s \\
& =\int_{0}^{+\infty} e^{-\frac{\varepsilon}{1-\varepsilon} s} e^{-s} \frac{s^{n-1}}{(n-1) !} \mathrm{d} s \\
& =(1-\varepsilon)^{n}
\end{aligned}
$$

The equality of the second line stems from an equality in distribution, since $S_{n+1}-Z_{i}$ has the same distribution as $S_{n}$ and $Z_{i}$ has the same distribution as $Z_{n+1}$, and the fact that $Z_{i}$ and $S_{n+1}-Z_{i}$ are independent. Taking $\left.\varepsilon=t \frac{\log (n)}{n} \in\right] 0,1[$, and using the standard inequality $\log (1-u) \leq-u$, for $u \in[0,1]$, we get

$$
\mathbb{P}\left(\delta_{i} \geq \varepsilon\right)=(1-\varepsilon)^{n}=\exp (n \log (1-\varepsilon)) \leq \exp (-n \varepsilon)=n^{-t}
$$

Proof of Theorem 3.6. The idea of the proof is to take the conditional probability with respect to a fixed realization $\mathbf{x}=\left(\mathbf{x}_{1}, \ldots, \mathbf{x}_{n}\right)$ of the random vector $\mathbf{X}$, then use the bound in Theorem 3.3, which is independent of $\mathbf{x}$, and finally integrate with respect to the uniform density on $\Omega^{n}$.

(i) We have

$$
\begin{aligned}
\mathbb{P}\left(\left\|u-\check{u}_{n}\right\|_{C\left(0, T ; L^{p}(\Omega)\right)} \geq \varepsilon^{\prime}\right) & =\frac{1}{|\Omega|^{n}} \int_{\Omega^{n}} \mathbb{P}\left(\left\|u-\check{u}_{n}\right\|_{C\left(0, T ; L^{p}(\Omega)\right)} \geq \varepsilon^{\prime} \mid \mathbf{X}=\mathbf{x}\right) d \mathbf{x} \\
& \leq \frac{1}{|\Omega|^{n}} \int_{\Omega^{n}} n^{-C_{2} q_{n}^{2 p-1} \beta} d \mathbf{x} \\
& =n^{-C_{2} q_{n}^{2 p-1} \beta}
\end{aligned}
$$

with

$$
\varepsilon^{\prime}=C_{1} T \exp (O(T))\left(\left(\beta \frac{\log (n)}{n}+\frac{\max \left(q_{n}^{-(p-1)}, q_{n}^{-p / 2}\right)}{n^{p / 2}}\right)^{1 / p}+\left\|K-\hat{K}_{n}\right\|_{L^{p}\left(\Omega^{2}\right)}+\left\|g-g_{n}\right\|_{L^{p}(\Omega)}+\tau\right) .
$$

Thus, (3.26) follows from the fact that the obtained bound in (3.7) is independent of the random choice of $\mathrm{x}$.

(ii) In view of (3.23), we can argue that

$$
\mathbb{P}\left(\left\|K-\hat{K}_{n}\right\|_{L^{p}\left(\Omega^{2}\right)}+\left\|g-g_{n}\right\|_{L^{p}(\Omega)} \geq \kappa\right) \leq \mathbb{P}\left(\left(C(p, q, s)+C\left(p, q, s^{\prime}\right)\right) \delta(n)^{\theta} \geq \kappa\right) .
$$

Taking $\kappa=\left(C(p, q, s)+C\left(p, q, s^{\prime}\right)\right)\left(t \frac{\log (n)}{n}\right)^{\theta}$, for $\left.t \in\right] 0, e[$, and applying Lemma 3.8, we deduce that

$$
\mathbb{P}\left(\left\|K-\hat{K}_{n}\right\|_{L^{p}\left(\Omega^{2}\right)}+\left\|g-g_{n}\right\|_{L^{p}(\Omega)} \geq \kappa\right) \leq n^{-t}
$$


Denote the events

$$
\begin{aligned}
& A_{1}:\left\{\left\|\check{v}_{n}-\check{u}_{n}\right\|_{C\left(0, T ; L^{p}(\Omega)\right)} \leq \varepsilon\right\} \\
& A_{2}:\left\{\left\|K-\hat{K}_{n}\right\|_{L^{p}\left(\Omega^{2}\right)}+\left\|g-g_{n}\right\|_{L^{p}(\Omega)} \leq \kappa^{\prime}\right\}
\end{aligned}
$$

and their complements $A_{i}^{c}$, where

$$
\varepsilon=C T \exp (O(T))\left(\left(\beta \frac{\log (n)}{n}+\frac{\max \left(q_{n}^{-(p-1)}, q_{n}^{-p / 2}\right)}{n^{p / 2}}\right)^{1 / p}+\tau\right)
$$

and $\kappa^{\prime}=C T \exp (O(T))\left(t \frac{\log (n)}{n}\right)^{\theta}$, with $C$ the largest constants among the one in claim (i) and $\left(C(p, q, s)+C\left(p, q, s^{\prime}\right)\right)$. Using the union bound, we get

$$
\begin{aligned}
\mathbb{P}\left(\left\|u-\check{u}_{n}\right\|_{C\left(0, T ; L^{p}(\Omega)\right)} \leq \varepsilon+\kappa^{\prime}\right) & \geq \mathbb{P}\left(\cap_{i=1}^{2} A_{i}\right)=1-\mathbb{P}\left(\cup_{i=1}^{2} A_{i}^{c}\right) \\
& \geq 1-\sum_{i=1}^{2} \mathbb{P}\left(A_{i}^{c}\right) \geq 1-\left(n^{-C_{2} q_{n}^{2 p-1} \beta}+n^{-t}\right),
\end{aligned}
$$

which yields the desired claim.

\subsection{Rate regimes}

A close inspection of the error bound in (3.27) (Thm. 3.6) reveals three contributions:

- Spatial discretization and edge sampling: the first contribution is materialized in the first term which scales as (see Rem. 3.4(ii))

$$
O\left(\left(\frac{\log (n)}{n}\right)^{1 / p}+\frac{\max \left(q_{n}^{-(1-1 / p)}, q_{n}^{-1 / 2}\right)}{n^{1 / 2}}\right) .
$$

This term represents the spatial discretization error when approximating the continuous evolution equation $(\mathcal{P})$ on the random inhomogeneous graph model $G_{q_{n}}(n, K)$ generated according to Definition 2.1 with the graphon $K$.

- Data approximation and node sampling: the second term is $O\left(\left(\frac{\log (n)}{n}\right)^{\theta}\right)$ which captures the error of approximating the initial data $g$ and the graphon $K$ on the grid of size $\delta(n)$ which concentrates around $\log (n) / n$. The presence of the error on $K$ is clearly tied to the nonlocal nature of the evolution equation on graphs. This approximation error depends on the regularity of $g$ and $K$, and the latter encodes the geometry/structure of the underlying graphs. The more regular $g$ and $K$ are, the faster the convergence rate.

- Time discretization: the last term, which is $O(\tau)$, is classical and corresponds to the time discretization error.

At this stage, one may wonder which of the first two terms dominate, or in other words, what are the different regimes exhibited by the convergence rate as a function of the problem parameters $\left(p, q, s, s^{\prime}\right)$. This is quite important as it will reveal which nonlocal $p$-Laplacian evolution problems are harder/easier to discretize 


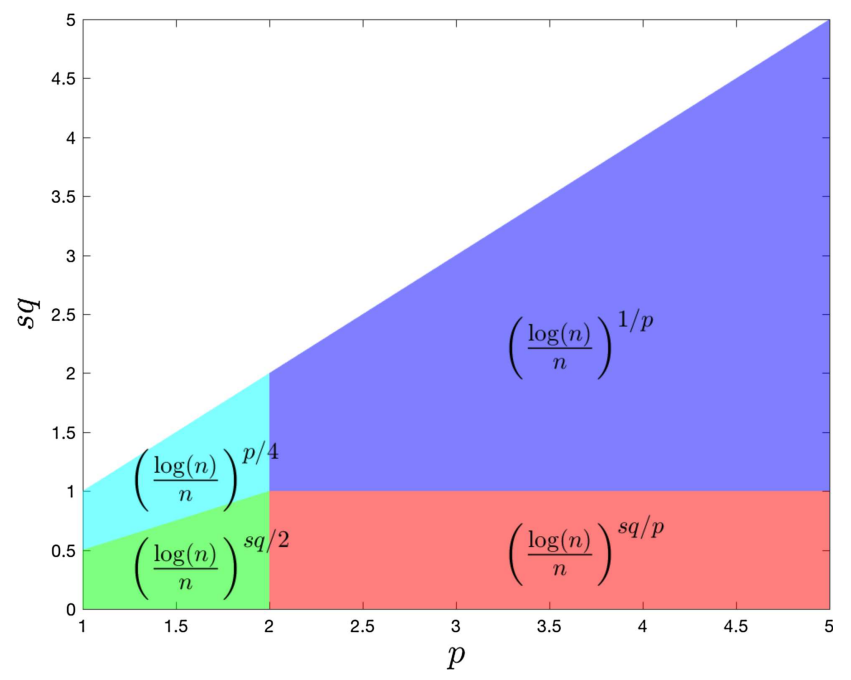

FiguRE 1. Different regimes according to the values of $p$ and $s$, and $q$.

by highlighting the role of each parameter, and for instance that of $p$ and the impact of nonlocality (i.e. graphon structure).

Toward this goal, we first make the error measure in (3.27) independent of $p$ and we choose to quantify the error in the classical $L^{2}(\Omega)$ norm. Consequently, thanks to the classical inequalities (3.9) and (A.9), as well as boundedness of the solutions, it is not difficult to see that

$$
\left\|u-\check{u}_{n}\right\|_{C\left(0, T ; L^{2}(\Omega)\right)}= \begin{cases}O\left(\left(\beta \frac{\log (n)}{n}\right)^{1 / p}+\frac{\max \left(q_{n}^{-(1-1 / p)}, q_{n}^{-1 / 2}\right)}{n^{1 / 2}}+\left(\frac{t \log (n)}{n}\right)^{\theta}+\tau\right), & p \in[2,+\infty[ \\ O\left(\left(\beta \frac{\log (n)}{n}\right)^{1 / 2}+\frac{\max \left(q_{n}^{-(p-1) / 2}, q_{n}^{-p / 4}\right)}{n^{p / 4}}+\left(\frac{t \log (n)}{n}\right)^{p \theta / 2}+\tau^{p / 2}\right) & p \in] 1,2],\end{cases}
$$

holds with probability at least $1-\left(n^{-C_{2} q_{n}^{2 p-1} \beta}+n^{-t}\right)$.

To make the rest of the discussion more concrete we will take $q_{n}=\log (n)^{-\delta /(2 p-1)}$, with $\delta \in[0,1[$, which covers both dense $(\delta=0)$ and non-dense $(\delta \in] 0,1[)$ graphs; see Remark 3.4(iii) and Proposition 2.4. Thus, we have

$$
\max \left(q_{n}^{-(1-1 / p)}, q_{n}^{-1 / 2}\right)= \begin{cases}O\left(\log (n)^{1 / 2}\right) & p \in[2,+\infty[ \\ O\left(\log (n)^{p / 4}\right) & p \in] 1,2]\end{cases}
$$

In turn, the second term in (3.31) is bounded by

$$
\left.\left(\frac{\log (n)}{n}\right)^{\min (p / 4,1 / 2)}, \forall p \in\right] 1,+\infty[.
$$

Without loss of generality ${ }^{10}$, we also suppose that $s=s^{\prime}$ and $q \leq p$ so that $\left.\left.\left.\left.\theta=s q / p \in\right] 0, q / p\right] \subset\right] 0,1\right]$. In this case, (3.31) reads

$$
\left\|u-\check{u}_{n}\right\|_{C\left(0, T ; L^{2}(\Omega)\right)}=O\left(\left(\frac{\log (n)}{n}\right)^{\min (1 / p, 1 / 2, s q / p) \min (p / 2,1)}+\tau^{\min (p / 2,1)}\right) .
$$

The term depending on $n$ then exhibits four different regimes as a function of $p, s$ and $q$ (see Fig. 1). Indeed, it is straightforward to see that it scales as

\footnotetext{
${ }^{10}$ This setting is true for many graphons, see, e.g. Remark 3.4(vi).
} 


$$
\begin{array}{llll}
\left(\frac{\log (n)}{n}\right)^{s q / p} & \text { for } & p \geq 2, & s q \in] 0,1], \\
\left(\frac{\log (n)}{n}\right)^{1 / p} & \text { for } & p \geq 2, & s q \in] 1, p], \\
\left(\frac{\log (n)}{n}\right)^{s q / 2} & \text { for } & p \in] 1,2], & s q \in] 0, p / 2], \\
\left(\frac{\log (n)}{n}\right)^{p / 4} & \text { for } & p \in] 1,2], & s q \in[p / 2, p] .
\end{array}
$$

In particular, the convergence rate shows a transition phenomenon at $p=2$. The rate increases with $p$ for $p \in] 2,+\infty[$ while it decreases with $p$ for $p \in] 1,2]$ and $s q \in[p / 2, p]$. As expected, the dependence of the rate on the initial data $g$ and graphon $K$ is more prominent as they become irregular, i.e. for smaller values of $s q$. For small $s q$ and $p \in] 1,2]$, the rate is independent of $p$.

\section{Appendix A.}

\section{A.1. A key deviation result}

The following lemma establishes a key deviation inequality for $\int_{0}^{T}\left\|Z_{n}(t)\right\|_{p, n} \mathrm{~d} t$ where $Z_{n}(\cdot)$ is a random process defined as

$$
Z_{n i}(t)=\frac{1}{n} \alpha_{i j}(t) \sum_{j=1}^{n}\left(\lambda_{i j}-\gamma_{i j}\right)
$$

where $\sup _{(i, j) \in[n]^{2}, t \in[0, T]}\left|\alpha_{i j}(t)\right|<+\infty$, and the $\lambda_{i j}$ 's are independent random variables such that $q_{n} \lambda_{i j}$ is Bernoulli with parameter $q_{n} \gamma_{i j}$, where $\sup _{i, j} \gamma_{i j}<+\infty$ and $q_{n}$ satisfies (A.2). It is obvious that this process covers that in (3.12) as a special case.

Lemma A.1. Let $Z_{n}(\cdot)$ be the random process defined in (A.1). Then, we have

(i) For $p \in[1,+\infty[, T>0$, there exists a positive constant $C$, such that for any $\beta>0$

$$
\mathbb{P}\left(\int_{0}^{T}\left\|Z_{n}(t)\right\|_{p, n} \mathrm{~d} t \geq \varepsilon\right) \leq n^{-C q_{n}^{2 p-1} \beta},
$$

with

$$
\varepsilon=T\left(\beta \frac{\log (n)}{n}+C_{3} \max \left(q_{n}^{-(p-1)}, q_{n}^{-p / 2}\right) \frac{1}{n^{p / 2}}\right)^{1 / p},
$$

where $C_{3}$ is a positive constant which will be explicit in the proof.

(ii) For $p \in[2,+\infty[$, suppose that there exists a positive constant $C$, such that for $t>0$

$$
\inf _{j \in[n]} \frac{1}{n} \sum_{i>j} \frac{\alpha_{i j}^{2}(t)}{q_{n}} \gamma_{i j}\left(1-q_{n} \gamma_{i j}\right) \geq C .
$$

Then,

$$
\mathbb{E}\left(\int_{0}^{T}\left\|Z_{n}(t)\right\|_{p, n}^{p} \mathrm{~d} t\right) \sim \frac{T}{n^{p / 2}} .
$$

To prove this lemma, we need the following deviation inequalities that we include for the reader convenience. Rosenthal's inequality [18]. Let $n$ be a positive integer, $\gamma \geq 2$ and $U_{1}, \ldots, U_{n}$ be $n$ zero mean independent random variables such that $\sup \mathbb{E}\left(\left|U_{i}\right|^{\gamma}\right)<\infty$. Then there exists a positive constant $C$ such that

$$
\mathbb{E}\left(\left|\sum_{i=1}^{n} U_{i}\right|^{\gamma}\right) \leq C \max \left(\sum_{i=1}^{n} \mathbb{E}\left(\left|U_{i}\right|^{\gamma}\right),\left(\sum_{i=1}^{n} \mathbb{E}\left(U_{i}^{2}\right)\right)^{\gamma / 2}\right) .
$$


Bernstein's inequality [23]. Let $n$ be a positive integer and $U_{1}, \ldots, U_{n}$ be $n$ zero mean independent random variables such that there exists a positive constant $M$ satisfying $\sup _{i \in[n]}\left|U_{i}\right| \leq M<\infty$. Then, for any $v>0$,

$$
\mathbb{P}\left(\sum_{i=1}^{n} U_{i} \geq v\right) \leq \exp \left(-\frac{v^{2}}{2\left(\sum_{i=1}^{n} \mathbb{E}\left(U_{i}^{2}\right)+v M / 3\right)}\right) .
$$

Proof of Lemma A.1. (i) Using the Jensen inequality, we have

$$
\mathbb{P}\left(\int_{0}^{T}\left\|Z_{n}(t)\right\|_{p, n} \mathrm{~d} t \geq \varepsilon\right) \leq \mathbb{P}\left(T^{p-1} \int_{0}^{T}\left\|Z_{n}(t)\right\|_{p, n}^{p} \mathrm{~d} t \geq \varepsilon^{p}\right) .
$$

Let us first recall that $q_{n} \lambda_{i j}$ are independent Bernoulli random variables with parameters $q_{n} \gamma_{i j}$. For the sake of simplicity, set, for $(i, j) \in[n]^{2}, Y_{n i} \stackrel{\text { def }}{=} \int_{0}^{T}\left|\frac{1}{n} \sum_{j=1}^{n} U_{n i j}(t)\right|^{p} \mathrm{~d} t$, where $U_{n i j}(t) \stackrel{\text { def }}{=} \alpha_{i j}(t)\left(\lambda_{i j}-\gamma_{i j}\right)$. We have

$$
I \stackrel{\text { def }}{=} \mathbb{P}\left(\int_{0}^{T}\left\|Z_{n}(t)\right\|_{p, n}^{p} \mathrm{~d} t \geq T^{1-p} \varepsilon^{p}\right)=\mathbb{P}\left(\frac{1}{n}\left(\sum_{i=1}^{n} Y_{n i}-\mathbb{E}\left(Y_{n i}\right)\right) \geq T^{1-p} \varepsilon^{p}-\frac{1}{n} \sum_{i=1}^{n} \mathbb{E}\left(Y_{n i}\right)\right) .
$$

It remains now to bound $\mathbb{E}\left(Y_{n i}\right)$. We distinguish the cases where $p \geq 2$ and $\left.p \in\right] 1,2[$.

$-p \geq 2$. Using the Rosenthal inequality with the independent according to $j$ zero-mean random variables $U_{n i j}(t)$, we have

$$
\begin{aligned}
\mathbb{E}\left(Y_{n i}\right) & =\frac{1}{n^{p}} \int_{0}^{T} \mathbb{E}\left(\left|\sum_{j=1}^{n} U_{n i j}(t)\right|^{p}\right) \mathrm{d} t \\
& \leq \frac{C_{1} T}{n^{p}} \sup _{t \in[0, T]} \max \left(\sum_{j=1}^{n} \mathbb{E}\left(\left|U_{n i j}(t)\right|^{p}\right),\left(\sum_{j=1}^{n} \mathbb{E}\left(U_{n i j}(t)^{2}\right)\right)^{p / 2}\right) .
\end{aligned}
$$

We have

$$
\begin{aligned}
\mathbb{E}\left(\left|U_{n i j}(t)\right|^{p}\right) & =q_{n}^{-p}\left|\alpha_{i j}(t)\right|^{p}\left|q_{n} \gamma_{i j}\left(1-q_{n} \gamma_{i j}\right)^{p}+\left(q_{n} \gamma_{i j}\right)^{p}\left(1-q_{n} \gamma_{i j}\right)\right| \\
& =q_{n}^{-(p-1)}\left|\alpha_{i j}(t)\right|^{p} \gamma_{i j}\left(1-q_{n} \gamma_{i j}\right)\left(\left(q_{n} \gamma_{i j}\right)^{p-1}+\left(1-q_{n} \gamma_{i j}\right)^{p-1}\right) .
\end{aligned}
$$

Taking $p=2$, we get

$$
\mathbb{E}\left(U_{n i j}(t)^{2}\right)=q_{n}^{-1} \alpha_{i j}^{2}(t) \gamma_{i j}\left(1-\gamma_{i j}\right) .
$$

Since $\sup _{(i, j) \in[n]^{2}, t \in[0, T]}\left|\alpha_{i j}(t)\right|<+\infty$, and $\gamma_{i j}$ is also bounded and $p$ being greater than 2 , there exists $C_{2}>0$, such that,

$$
\begin{aligned}
\max \left(\sum_{j=1}^{n} \mathbb{E}\left(\left|U_{n i j}(t)\right|^{p}\right),\left(\sum_{j=1}^{n} \mathbb{E}\left(U_{n i j}(t)^{2}\right)\right)^{p / 2}\right) & \leq C_{2} \max \left(n q_{n}^{-(p-1)}, n^{p / 2} q_{n}^{-p / 2}\right) \\
& \leq C_{2} \max \left(q_{n}^{-(p-1)}, q_{n}^{-p / 2}\right) n^{p / 2} .
\end{aligned}
$$

Therefore

$$
\frac{1}{n} \sum_{i=1}^{n} \mathbb{E}\left(Y_{n i}\right) \leq C_{1} C_{2} T \max \left(q_{n}^{-(p-1)}, q_{n}^{-p / 2}\right) n^{-p / 2} .
$$


- $p \in\left[1,2\left[\right.\right.$. Observe that by the mutual independence of the random variables $\left\{\lambda_{i j}\right\}_{(i, j) \in[n]^{2}}$, we deduce that $\left\{U_{n i j}(t)\right\}_{j=1}^{n}$ are independent and zero-mean random variables. Thus

$$
\mathbb{E}\left(\left(\sum_{j=1}^{n} U_{n i j}(t)\right)^{2}\right)=\operatorname{Var}\left(\sum_{j=1}^{n} U_{n i j}(t)\right)=\sum_{j=1}^{n} \mathbb{E}\left(U_{n i j}(t)^{2}\right)
$$

Therefore, applying the Jensen inequality to the concave function $x \mapsto x^{p / 2}$, we obtain

$$
\begin{aligned}
\mathbb{E}\left(Y_{n i}\right) \leq \frac{T}{n^{p}} \sup _{t \in[0, T]} \mathbb{E}\left(\left|\sum_{j=1}^{n} U_{n i j}(t)\right|^{p}\right) & \leq \frac{T}{n^{p}} \sup _{t \in[0, T]}\left(\mathbb{E}\left(\left(\sum_{j=1}^{n} U_{n i j}(t)\right)^{2}\right)\right)^{p / 2} \\
& =\frac{T}{n^{p}} \sup _{t \in[0, T]}\left(\sum_{j=1}^{n} \mathbb{E}\left(U_{n i j}(t)^{2}\right)\right)^{p / 2} \\
& =\frac{T}{n^{p}} \sup _{t \in[0, T]}\left(\sum_{j=1}^{n} \frac{\alpha_{i j}(t)^{2}}{q_{n}} \gamma_{i j}\left(1-q_{n} \gamma_{i j}\right)\right)^{p / 2} \\
& \leq \frac{C_{2} T}{q_{n}^{p / 2}} n^{-p / 2} \leq C_{2} T \max \left(q_{n}^{-(p-1)}, q_{n}^{-p / 2}\right) n^{-p / 2} .
\end{aligned}
$$

Altogether, we have shown that for any $p \geq 1$,

$$
\frac{1}{n} \sum_{i=1}^{n} \mathbb{E}\left(Y_{n i}\right) \leq C_{3} T \max \left(q_{n}^{-(p-1)}, q_{n}^{-p / 2}\right) n^{-p / 2}
$$

where $C_{3}=C_{2} \max \left(1, C_{1}\right)$.

Hence, setting $W_{n i}=Y_{n i}-\mathbb{E}\left(Y_{n i}\right)$ and $\kappa=T^{1-p} \varepsilon^{p}-C_{3} T \max \left(q_{n}^{-(p-1)}, q_{n}^{-p / 2}\right) n^{-p / 2}$, we have

$$
I \leq \mathbb{P}\left(\frac{1}{n} \sum_{i=1}^{n} W_{n i} \geq \kappa\right)
$$

Let $\varepsilon>0$ such that $\kappa>0$. Observe that the random variables $\left\{W_{n i}\right\}_{i=1}^{n}$ are independent, zero-mean, and obey:

- $\sup _{i \in[n]}\left|W_{n i}\right| \leq 2 \sup _{i \in[n]}\left|Y_{n i}\right| \leq C_{4} T$, since $\alpha_{i j}$ and $q_{n} \gamma_{i j}$ are both uniformly bounded.

- $\sum_{i=1}^{n} \mathbb{E}\left(W_{n i}^{2}\right)=\sum_{i=1}^{n} \operatorname{Var}\left(Y_{n i}\right) \leq \sum_{i=1}^{n} \mathbb{E}\left(Y_{n i}^{2}\right)$. Using the Jensen inequality with the function $x \mapsto x^{2}$, and replacing the exponent " $p$ " in inequality (A.2), by " $2 p$ " which is greater than 2 , we obtain

$$
\sum_{i=1}^{n} \mathbb{E}\left(W_{n i}^{2}\right) \leq \sum_{i=1}^{n} \mathbb{E}\left(Y_{n i}^{2}\right) \leq C_{5} T^{2} \max \left(q_{n}^{-(2 p-1)}, q_{n}^{-p}\right) \frac{1}{n^{p-1}}
$$


We are then in position to apply the Bernstein inequality to $\left\{W_{n i}\right\}_{i=1}^{n}$ according to the index $i$, whence we get, after some elementary algebra

$$
\begin{aligned}
\mathbb{P}\left(\frac{1}{n} \sum_{i=1}^{n} W_{n i} \geq \kappa\right) & \leq \exp \left(-\frac{n^{2} \kappa^{2}}{2\left(\sum_{i=1}^{n} \mathbb{E}\left(W_{n i}^{2}\right)+n \kappa C_{4} T / 3\right)}\right) \\
& \leq \exp \left(-\frac{C_{6}}{2} \min \left(q_{n}^{2 p-1}, q_{n}^{p}\right) \frac{n \kappa^{2}}{n^{-p} T^{2}+\kappa T}\right)
\end{aligned}
$$

Taking $\kappa=\beta T \frac{\log (n)}{n}>T n^{-p}$, for $p \geq 1$, we have after straightforward calculations

$$
\mathbb{P}\left(\frac{1}{n} \sum_{i=1}^{n} W_{n i} \geq \kappa\right) \leq \exp \left(-\frac{C_{6}}{4} \min \left(q_{n}^{2 p-1}, q_{n}^{p}\right) n \kappa / T\right)=n^{-\frac{C_{6}}{4} \min \left(q_{n}^{2 p-1}, q_{n}^{p}\right) \beta} .
$$

In turn,

$$
I \leq \mathbb{P}\left(\frac{1}{n} \sum_{i=1}^{n} W_{n i} \geq \kappa\right) \leq n^{-C \min \left(q_{n}^{2 p-1}, q_{n}^{p}\right) \beta} .
$$

For this choice of $\kappa$, observe that

$$
\begin{aligned}
\kappa=\beta T \frac{\log (n)}{n} & \Leftrightarrow T^{1-p} \varepsilon^{p}-C_{3} T \max \left(q_{n}^{-(p-1)}, q_{n}^{-p / 2}\right) n^{-p / 2}=\beta T \frac{\log (n)}{n} \\
& \Leftrightarrow \varepsilon=T\left(\beta \frac{\log (n)}{n}+C_{3} \max \left(q_{n}^{-(p-1)}, q_{n}^{-p / 2}\right) \frac{1}{n^{p / 2}}\right)^{1 / p} .
\end{aligned}
$$

Thus

$$
\mathbb{P}\left(\int_{0}^{T}\left\|Z_{n}(t)\right\|_{p, n} \mathrm{~d} t \geq \varepsilon\right) \leq n^{-C \min \left(q_{n}^{2 p-1}, q_{n}^{p}\right) \beta} .
$$

As $q_{n} \leq 1$ by (A.2) and $2 p-1 \geq p$ for $p \in \geq 1$, we obviously have $\min \left(q_{n}^{2 p-1}, q_{n}^{p}\right)=q_{n}^{2 p-1}$.

(ii) Recalling the notation in the proof of claim (i), we have

$$
\forall(i, j) \in[n]^{2}, \frac{1}{n} \sum_{i=1}^{n} Y_{n i}=\frac{1}{n} \int_{0}^{T} \sum_{i=1}^{n}\left|Z_{n i}(t)\right|^{p} \mathrm{~d} t=\frac{1}{n^{p+1}} \int_{0}^{T} \sum_{i=1}^{n}\left|\sum_{j=1}^{n} U_{n i j}(t)\right|^{p} \mathrm{~d} t .
$$

Thus, for $p \in[2,+\infty[$, applying the Jensen inequality and using (A.4), we have

$$
\begin{aligned}
\frac{1}{n} \sum_{i=1}^{n} \mathbb{E}\left(Y_{n i}\right) & =\frac{1}{n^{p+1}} \int_{0}^{T} \sum_{i=1}^{n} \mathbb{E}\left(\left|\sum_{j=1}^{n} U_{n i j}(t)\right|^{p}\right) \mathrm{d} t \\
& \geq \frac{1}{n^{p+1}} \int_{0}^{T} \sum_{i=1}^{n}\left(\mathbb{E}\left(\sum_{j=1}^{n} U_{n i j}(t)\right)^{2}\right)^{p / 2} \mathrm{~d} t \\
& =\frac{1}{n^{p+1}} \int_{0}^{T} \sum_{i=1}^{n}\left(\operatorname{Var}\left(\sum_{j=1}^{n} U_{n i j}(t)\right)\right)^{p / 2} \mathrm{~d} t
\end{aligned}
$$




$$
\begin{aligned}
& =\frac{1}{n^{p+1}} \int_{0}^{T} \sum_{i=1}^{n}\left(\sum_{j=1}^{n} \operatorname{Var}\left(U_{n i j}(t)\right)\right)^{p / 2} \mathrm{~d} t \\
& =\frac{1}{n^{p+1}} \int_{0}^{T} \sum_{i=1}^{n}\left(\sum_{j=1}^{n} \frac{\alpha_{i j}^{2}(t)}{q_{n}} \gamma_{i j}\left(1-q_{n} \gamma_{i j}\right)\right)^{p / 2} \mathrm{~d} t \\
& \geq C^{p / 2} T n^{-p-1} n^{p / 2+1} \geq \frac{C^{p / 2} T}{n^{p / 2}} .
\end{aligned}
$$

Combining this lower-bound with the upper-bounded (A.6), we get the claimed equivalence.

\section{A.2. Approximation theoretic results}

In an effort to make this paper more self-contained we briefly recall some results on functional spaces and approximation theory that our work relies on.

$L^{p}$ spaces embeddings. Since $|\Omega|=1$, we have the classical inclusion $L^{q}(\Omega) \subset L^{p}(\Omega)$ for $1 \leq p \leq q<+\infty$. More precisely

$$
\|F\|_{L^{p}(\Omega)} \leq\|F\|_{L^{q}(\Omega)} \leq\|F\|_{L^{\infty}(\Omega)} .
$$

We also have the reverse bound

$$
\|F\|_{L^{p}(\Omega)} \leq\|F\|_{L^{\infty}(\Omega)}^{1-q / p}\|F\|_{L^{q}(\Omega)}^{q / p}
$$

for any $1 \leq q<p<+\infty$.

Lipschitz spaces $\operatorname{Lip}\left(s, L^{q}\left(\Omega^{d}\right)\right)$ ([9], Ch. 2, Sects. 6 and 9). We introduce the Lipschitz spaces $\operatorname{Lip}\left(s, L^{q}\left(\Omega^{d}\right)\right)$, for $d \in\{1,2\}$, which contain functions with, roughly speaking, $s$ "derivatives" in $L^{q}\left(\Omega^{d}\right)$ ([9], Ch. 2, Sect. 9).

Definition A.2. For $F \in L^{q}\left(\Omega^{d}\right), q \in[1,+\infty]$, we define the (first-order) $L^{q}\left(\Omega^{d}\right)$ modulus of smoothness by

$$
\omega(F, h)_{q} \stackrel{\text { def }}{=} \sup _{\boldsymbol{z} \in \mathbb{R}^{d},|\boldsymbol{z}|<h}\left(\int_{\boldsymbol{x}, \boldsymbol{x}+\boldsymbol{z} \in \Omega^{d}}|F(\boldsymbol{x}+\boldsymbol{z})-F(\boldsymbol{x})|^{q} \mathrm{~d} \boldsymbol{x}\right)^{1 / q} .
$$

The Lipschitz spaces $\operatorname{Lip}\left(s, L^{q}\left(\Omega^{d}\right)\right)$ consist of all functions $F$ for which

$$
|F|_{\operatorname{Lip}\left(s, L^{q}\left(\Omega^{d}\right)\right)} \stackrel{\text { def }}{=} \sup _{h>0} h^{-s} \omega(F, h)_{q}<+\infty .
$$

We restrict ourselves to values $s \in] 0,1]$ as for $s>1$, only constant functions are in $\operatorname{Lip}\left(s, L^{q}\left(\Omega^{d}\right)\right)$. It is easy to see that $|F|_{\operatorname{Lip}\left(s, L^{q}\left(\Omega^{d}\right)\right)}$ is a semi-norm. $\operatorname{Lip}\left(s, L^{q}\left(\Omega^{d}\right)\right)$ is endowed with the norm

$$
\|F\|_{\operatorname{Lip}\left(s, L^{q}\left(\Omega^{2}\right)\right)} \stackrel{\text { def }}{=}\|F\|_{L^{q}\left(\Omega^{2}\right)}+|F|_{\operatorname{Lip}\left(s, L^{q}\left(\Omega^{d}\right)\right)} .
$$

The space $\operatorname{Lip}\left(s, L^{q}\left(\Omega^{2}\right)\right)$ is the Besov space $\mathbf{B}_{q, \infty}^{s}([9]$, Ch. 2, Sect. 10) which are very popular in approximation theory. In particular, $\operatorname{Lip}\left(1, L^{1}\left(\Omega^{d}\right)\right)$ contains the space $\mathrm{BV}\left(\Omega^{d}\right)$ of functions of bounded variation on $\Omega^{d}$, i.e. the set of functions $F \in L^{1}\left(\Omega^{d}\right)$ such that their variation is finite:

$$
V_{\Omega^{2}}(F) \stackrel{\text { def }}{=} \sup _{h>0} h^{-1} \sum_{i=1}^{d} \int_{\Omega^{d}}\left|F\left(\boldsymbol{x}+h e_{i}\right)-F(\boldsymbol{x})\right| \mathrm{d} \boldsymbol{x}<+\infty
$$

where $e_{i}, i \in\{1, d\}$ are the coordinate vectors in $\mathbb{R}^{d}$; see Ch. 2, Lemma 9.2 of [9]. Thus Lipschitz spaces are rich enough to contain functions with both discontinuities and fractal structure. 
Let us define the piecewise constant approximation of a function $F \in L^{q}\left(\Omega^{2}\right)$ (a similar reasoning holds of course on $\Omega$ ) on a partition of $\Omega^{2}$ into cells $\left.\left.\left.\left.\Omega_{n i j} \stackrel{\text { def }}{=}\{] x_{i-1}, x_{i}\right] \times\right] y_{j-1}, y_{j}\right]:(i, j) \in[n]^{2}\right\}$ of maximal mesh size $\delta \stackrel{\text { def }}{=} \max _{(i, j) \in[n]^{2}} \max \left(\left|x_{i}-x_{i-1}\right|,\left|y_{j}-y_{j-1}\right|\right)$,

$$
F_{n}(x, y) \stackrel{\text { def }}{=} \sum_{i, j=1}^{n} F_{n i j} \chi_{\Omega_{n i j}}(x, y), \quad F_{i j}=\frac{1}{\left|\Omega_{n i j}\right|} \int_{\Omega_{n i j}} F(x, y) \mathrm{d} x \mathrm{~d} y .
$$

Clearly, $F_{n}$ is nothing but the orthogonal projection of $F$ on the $n^{2}$-dimensional subspace of $L^{q}\left(\Omega^{2}\right)$ defined as

$$
\operatorname{Span}\left\{\chi_{\Omega_{n i j}}:(i, j) \in[n]^{2}\right\} .
$$

Lemma A.3. There exists a positive constant $C_{s}$, depending only on $s$, such that for all $F \in \operatorname{Lip}\left(s, L^{q}\left(\Omega^{d}\right)\right)$, $d \in\{1,2\}, s \in] 0,1], q \in[1,+\infty]$,

$$
\left\|F-F_{n}\right\|_{L^{q}\left(\Omega^{d}\right)} \leq C_{s} \delta^{s}|F|_{\operatorname{Lip}\left(s, L^{q}\left(\Omega^{d}\right)\right)} .
$$

Proof. Using the general bound ([9], Ch. 7, Thm. 7.3) for the error in spline approximation, and in view of Definition A.2, we have

$$
\left\|F-F_{n}\right\|_{L^{q}\left(\Omega^{d}\right)} \leq C_{s} \omega(F, \delta)_{q}=C \delta^{s}\left(\delta^{-s} \omega(F, \delta)_{q}\right) \leq C_{s} \delta^{s}|F|_{\operatorname{Lip}\left(s, L^{q}\left(\Omega^{d}\right)\right)} .
$$

An immediate consequence is the following result.

Lemma A.4. Assume that $\left.\left.F \in L^{\infty}\left(\Omega^{d}\right) \cap \operatorname{Lip}\left(s, L^{q}\left(\Omega^{d}\right)\right), d \in\{1,2\}, s \in\right] 0,1\right], q \in[1,+\infty]$, and let $\left.p \in\right] 1,+\infty[$. Then there exists a positive constant $C(p, q, s)$, depending on $p, q$ and $s$ such that

$$
\left\|F-F_{n}\right\|_{L^{p}\left(\Omega^{d}\right)} \leq C(p, q, s) \delta^{s \min (1, q / p)} .
$$

We have

$$
\begin{aligned}
& \left\|F-F_{n}\right\|_{L^{p}\left(\Omega^{d}\right)} \leq \\
& \begin{cases}\left\|F-F_{n}\right\|_{L^{q}(\Omega)} \leq C|F|_{\operatorname{Lip}\left(s, L^{q}(\Omega)\right)} \delta^{s}, & \text { if } q \geq p ; \\
\left\|F-F_{n}\right\|_{L^{\infty}\left(\Omega^{d}\right)}^{1-q / p}\left\|F-F_{n}\right\|_{L^{q}\left(\Omega^{d}\right)}^{q / p} \leq C\left(2\|F\|_{L^{\infty}(\Omega)}\right)^{1-q / p}|F|_{\operatorname{Lip}\left(s, L^{q}\left(\Omega^{d}\right)\right)}^{q / p} \delta^{s q / p} & \text { otherwise, }\end{cases}
\end{aligned}
$$

where we used (A.8) (resp. (A.9)) and Lemma A.3 in the first (resp. second) case.

Acknowledgements. This work was supported by the ANR grant GRAPHSIP. JF was partly supported by Institut Universitaire de France. JF and AE would like to acknowledge support within the EU grant No. 777826, the NoMADs project.

\section{REFERENCES}

[1] F. Andreu, J. Mazón, J. Rossi and J. Toledo, A nonlocal p-laplacian evolution equation with neumann boundary conditions. J. Math. Pures Appl. 90 (2008) 201-227.

[2] F. Andreu-Vaillo, J.M. Mazón, J.D. Rossi and J.J. Toledo-Melero, Nonlocal diffusion problems. In: Vol. 165 of Mathematical Surveys and Monographs. American Mathematical Society, Providence, RI (2010).

[3] B. Bollobás and O. Riordan, Metrics for sparse graphs, edited by S. Huczynska, J.D. Mitchell and C.M.E. Roney-Dougal. In: Surveys in Combinatorics 2009. London Mathematical Society Lecture Note Series. Cambridge University Press, Cambridge (2009) 211-288. 
[4] B. Bollobás and O. Riordan, Sparse graphs: metrics and random models. Random Struct. Algorithms 39 (2011) 1-38.

[5] B. Bollobás, S. Janson and O. Riordan, The phase transition in inhomogeneous random graphs. Random Struct. Algorithms 31 (2007) 3-122.

[6] C. Borgs, J. Chayes, L. Lovász, V. Sós, and K. Vesztergombi, Convergent sequences of dense graphs I: Subgraph frequencies, metric properties and testing. Adv. Math. 219 (2008) 1801-1851.

[7] C. Borgs, J. Chayes, L. Lovász, V. Sós and K. Vesztergombi, Limits of randomly grown graph sequences. Eur. J. Comb. 32 (2011) 985-999.

[8] A. Buades, B. Coll and J.-M. Morel, Neighborhood filters and PDEs. Numer. Math. 105 (2006) 1-34.

[9] R.A. DeVore and G.G. Lorentz, Constructive approximation. In: Vol. 303 of Grundlehren der Mathematischen. Springer-Verlag, Berlin Heidelberg (1993).

[10] A. Elmoataz, X. Desquesnes and O. Lezoray, Non-local morphological pdes and $p$-laplacian equation on graphs with pplications in image processing and machine learning. IEEE J. Sel. Top. Signal Process. 6 (2012) 764-779.

[11] A. Elmoataz, M. Toutain and D. Tenbrinck, On the $p$-laplacian and $\infty$-laplacian on graphs with applications in image and data processing. SIAM J. Imaging Sci. 8 (2015) 2412-2451.

[12] P. Erdös and A. Rényi, On the evolution of random graphs. Publ. Math. Inst. Hung. Acad. Sci. 5 (1960) 17-61.

[13] L.C. Evans, Partial Differential Equations. American Mathematical Society, Providence, RI (2010).

[14] E.N. Gilbert, Random graphs. Ann. Math. Stat. 30 (1959) 1141-1144.

[15] G. Gilboa and S. Osher, Nonlocal linear image regularization and supervised segmentation. Multiscale Model. Simul. 6 (2007) 595-630.

[16] G. Gilboa and S. Osher, Nonlocal operators with applications to image processing. Multiscale Model. Simul. 7 (2009) 10051028.

[17] Y. Hafiène, J. Fadili and A. Elmoataz, Nonlocal p-laplacian evolution problems on graphs. SIAM J. Numer. Anal. 56 (2018) 1064-1090.

[18] R. Ibragimov and S. Sharakhmetov, The exact constant in the Rosenthal inequality for random variables with mean zero. Theory Probab. App. 46 (2002) 127-132.

[19] D. Kaliuzhnyi-Verbovetskyi and G. Medvedev, The semilinear heat equation on sparse random graphs. SIAM J. Math. Anal. 49 (2017) 1333-1355.

[20] S. Kindermann, S. Osher and P.W. Jones, Deblurring and denoising of images by nonlocal functionals. Multiscale Model. Simul. 4 (2005) 1091-1115.

[21] L. Lovász, Large Networks and Graph Limits. American Mathematical Society 60 (2012).

[22] L. Lovász and B. Szegedy, Limits of dense graph sequences. J. Comb. Theory Ser. B 96 (2006) 933-957.

[23] P. Massart, Concentration inequalities and model selection. In: Vol. 1896 of Ecole d'Eté de Probabilités de Saint-Flour XXXIII - 2003. Springer Verlag, New York, NY (2007).

[24] G.S. Medvedev, The nonlinear heat equation on dense graphs. SIAM J. Math. Anal. 46 (2014) 2743-2766.

[25] G.S. Medvedev, The nonlinear heat equation on $W$-random graphs. Arch. Ration. Mech. Anal. 212 (2014) $781-803$.

[26] G.S. Medvedev, The continuum limit of the kuramoto model on sparse random graphs. Preprint arXiv:1802.03787 (2018).

[27] R.-D. Reiss, Approximate Distributions of Order Statistics with Applications to Nonparametric Statistics. Springer-Verlag, New York, NY (1989). 